\title{
Parenting and Self-Control Across Early to Late Adolescence: A Three-Level Meta-Analysis
}

Perspectives on Psychological Science 2019, Vol. 14(6) 967-1005 (c) The Author(s) 2019 Article reuse guidelines: sagepub.com/journals-permissions DOI: $10.1177 / 1745691619863046$ www.psychologicalscience.org/PPS (9SAGE

\author{
Jian-Bin Li ${ }^{1,2}{ }^{(D)}$, Yayouk E Willems ${ }^{3,4,5}$, F. Marijn Stok ${ }^{5}$, \\ Maja Deković, ${ }^{6}$ Meike Bartels ${ }^{3,4,7}$, and Catrin Finkenauer ${ }^{5}$ \\ ${ }^{1}$ Department of Early Childhood Education, The Education University of Hong Kong; ${ }^{2}$ Centre for \\ Child and Family Science, The Education University of Hong Kong; ${ }^{3}$ Department of Biological \\ Psychology, Vrije Universiteit Amsterdam; ${ }^{4}$ Amsterdam Public Health Institute, Amsterdam, the \\ Netherlands; ${ }^{5}$ Department of Interdisciplinary Social Sciences, Utrecht University; ${ }^{6}$ Department of \\ Clinical Child and Family Studies, Utrecht University; and ${ }^{7}$ Amsterdam Neuroscience, Amsterdam, \\ the Netherlands
}

\begin{abstract}
Self-control plays a significant role in positive youth development. Although numerous self-control challenges occur during adolescence, some adolescents control themselves better than others. Parenting is considered a critical factor that distinguishes adolescents with good self-control from those with poor self-control, but existing findings are inconsistent. This meta-analysis summarizes the overall relationship between parenting and self-control among adolescents aged 10 to 22 years. The analysis includes 191 articles reporting 1,540 effect sizes $(N=164,459)$. The results show that parenting is associated with adolescents' self-control both concurrently $(r=.204, p<.001)$ and longitudinally $(r=.157, p<.001)$. Longitudinal studies also reveal that adolescents' self-control influences subsequent parenting $(r=.155, p<.001)$. Moderator analyses show that the effect sizes are largely invariant across cultures, ethnicities, age of adolescents, and parent and youth gender. Our results point to the importance of parenting in individual differences in adolescent self-control and vice versa.
\end{abstract}

\section{Keywords}

parenting, parent-child relationship, self-control, adolescence, meta-analysis

Adolescence is a distinct and pivotal period of life with significant changes and growth at every level of functioning. What happens during adolescence has profound and long-lasting implications for youth's trajectories of economic security, health, well-being, and development into later life (Patton et al., 2016). It is also a sensitive period featured by the increasing prevalence of risk behaviors jeopardizing youth's physical and psychological functioning across their life course (Arnett, 1992, 1999; Casey, Jones, \& Hare, 2008; Steinberg, 2004). Consequently, numerous studies investigated what factors counteract adolescents' problem behaviors, and self-control is found to be such a factor (Caspi et al., 2016; Moffitt et al., 2011). Self-control-defined as one's ability to alter dominant responses to abide by social values and moral norms and to support the pursuit of long-term goals (Baumeister, Vohs, \& Tice, 2007; Tangney, Baumeister, \& Boone, 2004)_plays a key role in positive youth development and is widely studied across disciplines such as criminology, economics, public health, and neuroscience (Caspi et al., 2016; Figner et al., 2010; Hare, Camerer, \& Rangel, 2009). It helps adolescents get through a stage marked by a range of normative biological and social challenges (Crone \& Dahl, 2012), increases in risk taking and social reward-seeking behavior (Boyer, 2006; Smetana,

\footnotetext{
Corresponding Authors:

Jian-Bin Li, Department of Early Childhood Education, The Education University of Hong Kong, 10 Lo Ping Rd., Tai Po, New Territories, Hong Kong

E-mail: lijianbin@eduhk.hk

Yayouk E. Willems, Department of Biological Psychology, Vrije Universiteit, van der Boechorststraat 7-9, 1081 BT, Amsterdam, the Netherlands

E-mail: y.e.willems@vu.nl
} 
Campione-Barr, \& Metzger, 2006), and heightened emotional turmoil (Steinberg \& Morris, 2001).

For example, adolescents with higher levels of selfcontrol use less drugs and alcohol (Vazsonyi, TrejosCastillo, \& Huang, 2006), experience fewer emotional and behavioral problems (Finkenauer, Engels, \& Baumeister, 2005; J. B. Li, Delvecchio, Lis, Nie, \& Di Riso, 2015; Situ, Li, \& Dou, 2016; Vazsonyi \& Belliston, 2007), show more prosocial behavior (Nie, Li, \& Vazsonyi, 2016; PadillaWalker \& Christensen, 2011), and are more likely to do better academically than adolescents with lower levels of self-control (Duckworth \& Seligman, 2005; Galla \& Duckworth, 2015). Self-control generally continues to improve throughout adolescence in terms of neural and psychological development (Casey et al., 2008; Giedd, 2004; Romer, Duckworth, Sznitman, \& Park, 2010); however, some adolescents show better self-control than others. To enhance our understanding of how these individual differences in self-control come about during the unique period of adolescence, we sought through the current meta-analysis to examine the relation between parenting and self-control across adolescence by considering the joint contribution of adolescents and parents to adolescent development.

Theorists agree that parenting is an important factor associated with individual differences in self-control (Bridgett, Burt, Edwards, \& Deater-Deckard, 2015; Gottfredson \& Hirschi, 1990; Kochanska, 1993; Kopp, 1982; Sameroff, 2010). More specifically, prior studies have found that positive parenting (e.g., monitoring, consistent discipline, parental warmth and support, positive control, authoritative parenting) and a strong parentchild relationship (e.g., secure attachment, close parentchild bonding, high quality of relationship) are related to better self-control. Conversely, negative parenting (e.g., inconsistent discipline, harsh parenting, coercive parenting, physical punishment, negative control, authoritarian parenting) and a weak parent-child relationship are associated with lower self-control in early and middle childhood (see Davis, Bilms, \& Suveg, 2017; Karreman, van Tuijl, van Aken, \& Deković, 2006; Pallini et al., 2018). However, studies extending this work to adolescence yield mixed findings. Whereas some studies have reported robust cross-sectional and longitudinal associations between parenting and self-control throughout adolescence (Hay, 2001; Hope, Grasmick, \& Pointon, 2003; Özdemir, Vazsonyi, \& Çok, 2013; Vazsonyi \& Belliston, 2007), others have reported only significant concurrent associations (Baardstu, Karevold, \& von Soest, 2017; Craig, 2016; Moilanen, Rasmussen, \& PadillaWalker, 2015; Pallini et al., 2018). Moreover, some studies have found significant associations for some parenting dimensions but not others (Finkenauer et al., 2005; Vazsonyi, Jiskrova, Ksinan, \& Blatny, 2016). Thus, previous findings regarding the magnitude and direction of the association between parenting and self-control in adolescence are not conclusive.

These inconsistent findings might be explained by the notion that parenting is most strongly associated with self-control during early and middle childhood and less in other developmental periods (Gottfredson \& Hirschi, 1990; Hay \& Meldrum, 2016; Kochanska, 1993; Kopp, 1982; Meldrum, Young, Hay, \& Flexon, 2012; Vazsonyi \& Jiskrova, 2017). During early and middle childhood, children rely mostly on their parents for decision making, behavior guidance, and emotion regulation, and parents support children with their selfregulatory capacities by providing an environment in which they assist their children in regulating inner feelings and behaviors (Kopp, 1982; Sameroff, 2010). When entering the teenage years, adolescents transit into a phase with relative independence as they gradually desire more autonomy from parents: Adolescents interact less with parents and more with peers and are more likely to negotiate with parents about social customs and/or conventions instead of merely following parents' guidance (Steinberg \& Silk, 2002). Thus, in the teenage years the influence of parenting on the development of self-control may decrease (Farley \& Kim-Spoon, 2014; Hay \& Meldrum, 2016; Tiberio et al., 2016).

However, although adolescents may feel they are independent and responsible individuals, parents generally remain important figures adolescents turn to for emotional and financial support when needed (Buist, Deković, Meeus, \& van Aken, 2002; J. B. Li, Delvecchio, Miconi, Salcuni, \& Di Riso, 2014). These social changes and developmental transitions in adolescence yield an important question: Is parenting still important for selfcontrol during adolescence? Asking this question is all the more important because scientists increasingly recognize that developmental and growth processes that have their beginning in early adolescence continue into the 20s (Sawyer, Azzopardi, Wickremarathne, \& Patton, 2018). In addition, as societal changes lead to delays in adopting adult roles (e.g., life expectancy, longer educational trajectories), research calls for extending our conceptualization of adolescence as ranging from early adolescence to the early 20s (Arroyo, Payne, Brown, \& Manning, 2013; Sawyer et al., 2018). In light of these considerations, researchers have pointed out that the adolescent period covers "a greater proportion of the life course with greater relevance for human development than ever before" (Patton et al., 2018, p. 458). Thus, it is crucial to take stock of the empirical findings and inform the field about the association between parenting and self-control across the course of adolescence.

The current meta-analysis adds to previous metaanalyses in several important ways. Previous metaanalyses regarding the association between parenting and self-control have focused either on young children 
(Davis et al., 2017; Karreman et al., 2006) or on specific parenting practices or indicators such as parent-child synchrony (Davis et al., 2017) and attachment-security status (Pallini et al., 2018). Moreover, existing metaanalyses have failed to take possible reciprocal effects of self-control on subsequent parenting into account (Sameroff, 2010). It is especially important during adolescence to consider the effects of adolescent self-control on parenting, which may play a particularly important role in promoting and maintaining its continuity across adolescence. Indeed, it may well be that self-control in early adolescence evokes responses from parents that reinforce the child's tendencies over time (e.g., Caspi \& Roberts, 2001). Finally, existing studies have not applied three-level analyses, a novel technique that better distinguishes variance at the sample, within-study, and between-study levels to provide a more accurate estimate of the results (Assink \& Wibbelink, 2016), such as taking into account dependency between different effect sizes extracted from the same study without losing the rich information of a study that contains multiple effect sizes (M. W. L. Cheung, 2014; Van den Noortgate, LópezLópez, Marín-Martínez, \& Sánchez-Meca, 2013).

To our knowledge, a comprehensive review synthesizing empirical studies on the association between broad types of parenting and self-control among adolescents is still lacking. This is crucial because the interplay between individuals and their social environments is at the core of the development of self-control, especially across the adolescent period. Adolescents increasingly become active agents of their own development, and their levels of self-control are likely to evoke responses from their parents, generating a reciprocal influence of persons and environments (Eisenberg et al., 2005; Sameroff, 2010; Tiberio et al., 2016). We wanted to clarify whether parenting is associated with self-control across adolescence and to identify factors that influence this association; consequently, this preregistered meta-analysis ${ }^{1}$ aims to answer (a) whether parenting is important to the self-control of adolescents aged 10 to 22 years, ${ }^{2}$ (b) whether adolescents' selfcontrol influences subsequent parenting, and (c) whether theoretical (e.g., types of parenting, age, culture, parent and adolescent gender) and methodological (e.g., report informant, consistency of report informant, and study design) factors included in previous meta-analyses about self-control moderate the magnitude of the association between parenting and self-control in adolescence. Investigating these questions allows us to aggregate diverse individual study results to identify the overall mean effect and examine the role of possible moderators on the magnitude of this effect. Doing so generates insights about selfcontrol development over the course of adolescence and elucidates gaps that should be given attention in future research aiming to understand individual differences in this important capacity.

\section{Conceptualization of Self-Control}

Research on self-control spans the social and behavioral sciences (Duckworth \& Steinberg, 2015). The nomenclature of self-control varies by theoretical tradition, with social psychologists and criminologists referring more often to self-control (e.g., Gottfredson \& Hirschi, 1990; Tangney et al., 2004) and developmental psychologists referring to concepts such as self-regulation and effortful control (e.g., Bridgett et al., 2015; Eisenberg et al., 2003; Eisenberg et al., 2005; Kopp, 1982).

Although there is considerable dissent in the literature about how to label or define self-control, existing theories and findings generally agree that self-control, self-regulation, and effortful control tap into the same capacity. The common thread running through these concepts is the involvement of voluntary self-governance, an ability one consciously uses to manage one's cognition, emotion, and behavior (Bridgett et al., 2015; Duckworth \& Kern, 2011; Nigg, 2017). Evidence from various aspects supports this view. First, studies applying factor-structure analyses combining tasks attributed to different conceptualizations of self-control are best presented by a single-factor model (Allan, Hume, Allan, Farrington, \& Lonigan, 2014; Allan \& Lonigan, 2011). Second, a metaanalysis that summarized the convergence of a number of self-control measures derived from different theories, perspectives, and approaches revealed that these measures are moderately convergent (Duckworth \& Kern, 2011). In addition, neuroscientific research showed overlapping neural substrates associated with these concepts (Fan, Flombaum, McCandliss, Thomas, \& Posner, 2003; Garavan, Ross, Murphy, Roche, \& Stein, 2002). Therefore, in this study, we included self-control as well as these analogous terms, referring to them overall as "self-control," as done by prior meta-analyses on selfcontrol (Davis et al., 2017; De Ridder, Lensvelt-Mulders, Finkenauer, Stok, \& Baumeister, 2012; Karreman et al., 2006; Pallini et al., 2018; Vazsonyi, Mikuška, \& Kelley, 2017).

\section{The Association Between Parenting and Self-Control}

Parenting is a multifaceted construct containing various terms reflecting different aspects of parenting processes. In this research, we mainly focused on three broad dimensions of parenting (i.e., positive parenting practices, negative parenting practices, and parent-child relationships) in relation to adolescent self-control. 
Positive parenting refers to parental behaviors that reflect positive control and warmth, such as parental warmth, monitoring, supervision, consistent discipline, parental support, and authoritative parenting (Darling \& Steinberg, 1993; T. G. O'Connor, 2002). Negative parenting refers to behaviors that reflect negative control and hostility, such as harsh parenting, inconsistent discipline, coercive punishment, authoritarian parenting, and permissive parenting (Darling \& Steinberg, 1993; T. G. O'Connor, 2002). Parent-child relationships refer to children's emotional bonds with their parents (Cassidy, 1994); this construct is also often labeled parent-child attachment (Bowlby, 1969) or parent-child bonding (Gottfredson \& Hirschi, 1990) across the literature.

The reasons we concentrate on these three broad types of parenting are threefold. First, the parenting literature has primarily focused on two broad categories of parenting, namely parenting behavior and practices (Darling \& Steinberg, 1993), which is further divided into positive and negative parenting practices (T. G. O'Connor, 2002), and emotional relationships or bonds between parents and children (Belsky, 1984; Bowlby, 1969). Second, these three parenting categories correspond to existing theories from various disciplines. For example, the general theory of crime postulates that self-control is nurtured by positive parenting practices (monitoring, consistent discipline) and a close parent-child relationship, whereas negative parenting practices (excessive punishment, permissive parenting) and a weak parent-child relationship undermine self-control development (Gottfredson \& Hirschi, 1990). The process model (Belsky, 1984) also suggests that parenting and parent-child relationships are different constructs within the family system that affect child development (Belsky, 1984). Third, as shown in Table 1, we have identified a host of specific parenting terms, with some (e.g., conflict, monitoring, authoritative) much more frequently used to examine the parenting-self-control association than others (e.g., neglect, overprotection, alienation). An advantage of focusing on the three broad types of parenting dimensions is that it allows us to group many relevant studies together, ensuring sufficient statistical power. If we focused on each specific term separately, it would not be possible to conduct moderation analyses because many terms would appear in the literature only a few times (e.g., neglect, overprotection, alienation) and statistical power would be low. Hence, in this study we focused on the three types of parenting dimensions, a strategy adopted by prior meta-analyses on parenting and child outcomes (e.g., Davis et al., 2017; Karreman et al., 2006; Pallini et al., 2018; Slagt, Dubas, Deković, \& van Aken, 2016).
Table 1. Details on Parenting Dimension

\begin{tabular}{|c|c|c|c|}
\hline \multirow[b]{2}{*}{ Parenting term } & \multicolumn{3}{|c|}{ Number of effect sizes $(N=1,540)$} \\
\hline & $\begin{array}{l}\text { Positive } \\
\text { parenting }\end{array}$ & $\begin{array}{l}\text { Negative } \\
\text { parenting }\end{array}$ & $\begin{array}{l}\text { Parent-child } \\
\text { relationship }\end{array}$ \\
\hline Authoritative & 139 & & \\
\hline Monitoring & 137 & & \\
\hline Support & 133 & & \\
\hline Responsive discipline & 95 & & \\
\hline Warmth & 81 & & \\
\hline Supervision & 53 & & \\
\hline Positive control & 46 & & \\
\hline Involvement & 33 & & \\
\hline Positive expressivity & 25 & & \\
\hline Sensitivity & 24 & & \\
\hline Acceptance & 21 & & \\
\hline Autonomy & 17 & & \\
\hline Cohesion & 6 & & \\
\hline Conflict & & 117 & \\
\hline Authoritarian & & 97 & \\
\hline $\begin{array}{l}\text { Psychological negative } \\
\text { control }\end{array}$ & & 81 & \\
\hline Harshness & & 78 & \\
\hline Permissive & & 44 & \\
\hline Abuse & & 26 & \\
\hline Rejection & & 23 & \\
\hline Hostility & & 16 & \\
\hline Withdrawal & & 14 & \\
\hline Negativity & & 10 & \\
\hline Coercion & & 7 & \\
\hline Attachment & & & 108 \\
\hline Relationship & & & 47 \\
\hline Bond & & & 21 \\
\hline Closeness & & & 16 \\
\hline Communication & & & 12 \\
\hline Trust & & & 8 \\
\hline Alienation & & & 5 \\
\hline
\end{tabular}

Note: The associations between these dimensions and self-control are presented in Table S1 in the Supplemental Material available online.

\section{The influence of parenting on self-control}

Parents who use positive parenting strategies provide clear standards for behavior (Sroufe, 1996), monitor and discipline their children's undesirable behavior timely and consistently (Gottfredson \& Hirschi, 1990), and guide them to work through problems themselves (Putnam, Spritz, \& Stifter, 2002; Strand, 2002), all of which help them gradually internalize others' rules and expectations of what are appropriate behaviors and may foster the development of self-control in adolescents. Many studies linked positive parenting with youth's self-control, generally finding that positive parenting relates to good self-control development in 
adolescents (e.g., Finkenauer et al., 2005; Hay, 2001; Hope et al., 2003; Özdemir et al., 2013; Vazsonyi \& Belliston, 2007). However, it warrants attention that some inconsistent findings have emerged in terms of significance and magnitude. For example, Baardstu et al. (2017) found no significant longitudinal associations between positive parenting and self-control over the course of early adolescence.

Parents who use negative parenting strategies are likely to deprive youths of opportunities to figure out self-control strategies independently (Grolnick, McMenamy, \& Kurowski, 1999), provide youths with little or no guidance to self-regulate when needed (Baumrind, 1991), monitor and discipline youths' undesirable behavior inconsistently or ineffectively (Gottfredson \& Hirschi, 1990), and create a stressful family environment that jeopardizes children's internalization of social rules (Kochanska, Aksan, \& Koenig, 1995; Silverman \& Ragusa, 1990). Negative parenting therefore provides a context that hampers the development of self-control in adolescence. Consistent with this suggestion is prior research that has generally found that negative parenting is related to low self-control (e.g., Brody \& Ge, 2001; N. W. T. Cheung \& Cheung, 2010; Feldman \& Wentzel, 1990; Hallquist, Hipwell, \& Stepp, 2015; Simons, Simons, Chen, Brody, \& Lin, 2007). Again, however, the evidence is inconsistent. For instance, prior research found that mothers' authoritarian and permissive parenting style and fathers' authoritarian and permissive parenting style were not significantly related to their adolescent children's self-control (Jabagchourian, Sorkhabi, Quach, \& Strage, 2014).

For parent-child relationships, parents who successfully establish close bonds help children to develop better emotional regulation ability, which lays the foundation for the further development of self-control (Bowlby, 1969; Cassidy, 1994; Kopp, 1982; Pallini et al., 2018). A close parent-child relationship (in some studies defined as secure parent-child attachment) ${ }^{3}$ during adolescence serves as the foundation for parents to monitor, recognize, and discipline adolescents' behavior (Gottfredson \& Hirschi, 1990) and, in turn, encourages children to share information about their daily activities with their parents (Kerr \& Stattin, 2000; Parke, 2004). A close parent-child relationship thereby provides a context that is conducive to parental scaffolding and the teaching of self-control. Consistent with this suggestion are numerous studies that have found that secure attachment is associated with better self-control (e.g., Alvarez-Rivera \& Fox, 2010; J. B. Li et al., 2015; Nie et al., 2016; Wills, Gibbons, Gerrard, Murry, \& Brody, 2003; You \& Kim, 2016). Nevertheless, the strength of the association varied considerably. Some studies found that the relation between parent-child relationships and self-control was close to zero (e.g., Jones, Lynam, \& Piquero, 2015), some found the relation to be significant but small (e.g., Walters \& DeLisi, 2013), and some found the relation to be significant with a medium effect size (e.g., Watts \& McNulty, 2016).

\section{The influence of self-control on parenting}

Adolescents are not passive recipients to parenting behaviors. Over the course of adolescence, their behaviors increasingly influence parenting behaviors. As such, adolescent development can be understood as a transactional or reciprocal process in which environmental factors (e.g., parenting) affect the development of adolescents (e.g., self-control) while an adolescent's behavior can also evoke certain reactions from his or her environment (for details, see the transactional model of development, Sameroff, 2010; social cognitive theory of personality, Bandura, 1999; genotype-environment correlation, Plomin, DeFries, \& Loehlin, 1977; and ecological-systems theory, Bronfenbrenner, 1979).

In parent-child dyads, parenting behaviors (e.g., parental trust and warmth) are related to parents' knowledge about their children (Kerr, Stattin, \& Trost, 1999). When parents know their children can exert self-control, resist temptations, and regulate their own behavior, parents are likely to trust their children, grant more autonomy, and respond positively to their behavior (Buyukcan-Tetik, Finkenauer, Siersema, Vander Heyden \& Krabbendam, 2015). Prior studies involving adolescents and parents have found that adolescents with high levels of self-control elicit trust and warmth from their parents (Bradley \& Corwyn, 2007; BuyukcanTetik et al., 2015; Tiberio et al., 2016). However, longitudinal research conducted among adolescents reveals nonsignificant effects of self-control on parenting over time (Eisenberg et al., 2005; Moilanen et al., 2015).

Likewise, children with good self-control are less impulsive and restless and engage in more socially desirable behavior, which may facilitate parents' relationship with them (Meldrum, Young, Hay, \& Flexon, 2012). Although some studies have supported this relation in adolescents (Bradley \& Corwyn, 2007; Otterpohl \& Wild, 2015), other studies failed to find such associations in adolescent samples (Meldrum et al., 2012). Conversely, adolescents with poor self-control are likely to engage in delinquent behavior and succumb to temptation, which may spur parents to assert their power by using harsh or coercive practices to teach their children a lesson and ostensibly prevent the occurrence of the behavior in the future (Karreman et al., 2006; Kochanska et al., 1995; Silverman \& Ragusa, 1990). For instance, prior research has found that adolescents with poor 
self-control are likely to elicit harsh parenting 1 year after baseline (Brody \& Ge, 2001). However, inconsistent findings also emerge: Moilanen et al. (2015) found that adolescents' self-control negatively predicts mothers' but not fathers' authoritarian parenting.

\section{Potential Moderators}

As shown above, the findings of the association of adolescent self-control and parenting are not consistent. This implies that the association between parenting and adolescent self-control may be moderated by several other factors, such as culture, age, and gender. A number of potential moderators are listed below.

\section{Theoretical moderators}

Type of parenting. Parenting is commonly categorized as positive and negative in terms of control and warmth (Darling \& Steinberg, 1993; T. G. O'Connor, 2002), suggesting that positive and negative parenting include both emotional (e.g., affection, warmth) and behavioral (e.g., monitoring and control) components. A close relationship refers to emotional bonding and thus seems to solely represent the emotional component. Although a close emotional bond is important to the development of selfcontrol, it is not enough to instill children with selfcontrol without consistent discipline and appropriate monitoring (Gottfredson \& Hirschi, 1990). This implies that the three categories of parenting may play a different role in the development of adolescent self-control. Therefore, in this study, we explored whether the three types of parenting relate differently to self-control over the course of adolescence.

Age. As adolescents develop, they become more independent, gain more autonomy from parents, negotiate more about social conventions, and become less attached to their family (Steinberg \& Silk, 2002), suggesting that the association of parenting and self-control is likely to decrease over the course of adolescence. Recent research examined the influence of parenting on effortful control from early childhood to early adolescence, revealing that the effects of parenting practices (both positive and negative practices) decreased as children grew older (Tiberio et al., 2016). Moreover, in the study by Vazsonyi and Belliston (2007), the associations between positive parenting (i.e., closeness, support, monitoring) and low selfcontrol were lower among U.S. college students $(r=$ $-.104,-.166$, and -.117 , respectively) than those among U.S. urban $(r=-.212,-.248$, and -.219 , respectively) and rural $(r=-.185,-.326$, and -.132 , respectively) high school students. These findings, coinciding with theories on the development of autonomy in adolescence (e.g., Collins \& Steinberg, 2006), led us to hypothesize that the association between parenting and self-control would diminish as adolescents become older.

Culture. Prior research has revealed cross-cultural differences in parenting. For instance, Chinese parents are thought to use a more authoritarian style (or harsh parenting) than Western parents (Chao, 1994; Ng, Pomerantz, \& Deng, 2014). However, scholars argue that parenting is closely dependent on cultural contexts and therefore any type of parenting, no matter whether it is positive or negative, should be effective in socializing children in a given culture (Fu \& Markus, 2014; for an overview, see Smetana, 2017). Some cross-cultural studies directly compared the association between parenting and self-control in adolescents from different cultural and ethnical backgrounds but yielded mixed evidence. For instance, Vazsonyi and colleagues found that the association between positive parenting (e.g., closeness and monitoring) was significantly related to self-control in Swiss, Dutch, and Hungarian adolescents but not in Slovenian or Japanese (for monitoring only) adolescents and concluded such inconsistencies might be due to cultural differences in parenting (Vazsonyi \& Belliston, 2007; Vazsonyi et al., 2006). In a study performed among Czech adolescents, Vazsonyi et al. (2016) found a positive relation between parental monitoring and self-control for Roma but not for non-Roma adolescents. Conversely, J. B. Li and colleagues (2015) found that the association between attachment to parents and self-control was largely invariant between Chinese and Italian adolescents. Given these findings, we explored whether culture moderates the parenting-selfcontrol relation.

Culture contains multiple dimensions, and individualism is one of the most frequently used variables to define cultures (Oyserman, Coon, \& Kemmelmeier, 2002). According to Hofstede (2001), some countries are more individualistic than others; thus, he developed an "individualism index" to reflect the levels of individualism of a country. In this sense, culture is treated as a continuum instead of a dichotomous category (e.g., Eastern vs. Western; independent vs. dependent). Moreover, we were aware that a number of studies on selfcontrol and parenting involved several ethnicities within their own country that may be not entirely mapped onto a country's levels of individualism (e.g., Asians residing in the United States). Thus, we used both Hofstede's individualism score and ethnicity to capture the role of culture. In this study, we explored whether the individualism score of the country in which the samples were recruited and adolescent ethnicities would moderate the parenting-self-control association. 
Adolescent gender. Research on gendered socialization (Gerson, 1985; Hagan, Simpson, \& Gillis, 1987; HayslettMcCall \& Bernard, 2002) suggests that the processes shaping self-control may differ by gender. For instance, parents may tolerate certain behaviors (e.g., hanging out with friends at night) from boys that would be quickly curtailed if displayed by girls. This suggests that the influence of parenting on self-control could differ between boys and girls. Some studies have found that the associations between parenting and self-control were larger for girls than for boys (e.g., Evans, Simons, \& Simons, 2012; Larsen et al., 2012; Mandara \& Pikes, 2008). However, another line of work suggests that although parents may use different strategies to educate boys and girls, the effectiveness of parental socialization on children's development of self-control is comparable (Beaver, Wright, \& DeLisi, 2007; Chapple, Vaske, \& Hope, 2010; J. B. Li et al., 2015; Lynskey, Winfree, Esbensen, \& Clason, 2000). In this study, we explored whether the association between parenting and self-control differed as a function of the proportions of boys and girls in the study sample.

Parent gender. Mothers are traditionally considered to be the main caregiver in the home and the most important socialization agent (Buist et al., 2002; Munroe, Munroe, Westling, \& Rosenberg, 1997; Song, Thompson, \& Ferrer, 2009). Yet some theories suggest that fathers and mothers are equally important to the socialization of children (Gottfredson \& Hirschi, 1990; Lamb, 2010). Moreover, it is theorized that despite the traditional role of mothers in the family, fathers also play a significant role in children's adjustment, including helping children develop control of their misbehavior (Lamb \& TamisLeMonda, 2004). However, empirical evidence for these suggestions is mixed. In some studies, the association between parenting and self-control appears stronger for maternal parenting (Intravia, Jones, \& Piquero, 2012; Patock-Peckham, Cheong, Balhorn, \& Nagoshi, 2001) or for paternal parenting (Feldman \& Wentzel, 1990; Morris \& Age, 2009), whereas some studies find a similar magnitude for both maternal and paternal parenting (e.g., J. B. Li et al., 2015; Nie et al., 2016; Özdemir et al., 2013). Given such disparities, we explored whether the parenting-selfcontrol association varied as a function of the proportions of mothers and fathers in the study sample.

\section{Metbodological moderators}

Report informants. Studies use a variety of methods to examine the relationship between parenting and selfcontrol. Some studies use self-report measures, some use other informants (e.g., parent-report, teacher-report), and others even use observational and behavioral methods. Results on the parenting-self-control association may vary across informants because family members as well as teachers and observers may have different experiences or views regarding parent-child interactions and adolescent self-control (Duckworth \& Kern, 2011; Lanz, Scabini, Vermulst, \& Gerris, 2001). In the current study, we explored whether the relation between parenting and self-control was different among different report informants.

Consistency of report informants. We further examined whether the consistency of report informants across constructs may moderate the link between parenting and self-control. Hypothetically, when the two constructs are assessed by the same informant (especially using selfreport measures), their correlation is likely to be higher than when the two constructed are assessed by different informants (Willems et al., 2018). In addition, research has found that the correlation between self-reports and otherreports on personality questionnaires is higher than the correlation between self-reports and behavioral tests (Duckworth \& Kern, 2011; Harden et al., 2017; Meyer et al., 2001). In light of this evidence, we explored whether the association between parenting and self-control is stronger when the two constructs were assessed using the same (i.e., consistent) rather than different (i.e., inconsistent) informants.

Study design. Both cross-sectional and longitudinal designs are used to test the association between selfcontrol and parenting, but differences in the magnitude of concurrent versus longitudinal associations are not well quantified. Such comparisons have been done in other meta-analyses focusing on the link between selfcontrol and deviance, with some studies revealing larger effect sizes for cross-sectional than for longitudinal study designs (Pratt \& Cullen, 2000) but others finding no significant differences between designs (Vazsonyi et al., 2017). In this meta-analysis, we tested whether the association between parenting and self-control would be different in magnitude for cross-sectional versus longitudinal studies. Using longitudinal studies, we also explored whether the influence of parenting on later self-control differed from the influence of adolescent self-control on later parenting. This examination allowed us to pit the effect of parenting on self-control and the effect of selfcontrol on parenting against each other.

\section{Method}

The Preferred Reporting Items for Systematic Reviews and Meta-Analyses (PRISMA) checklist (Moher et al., 2015) was used as a guideline for the setup of this metaanalysis. Furthermore, to facilitate transparency (Lakens, Hilgard, \& Staaks, 2016), the aim and hypotheses of this meta-analysis were preregistered at AsPredicted (https:// 
aspredicted.org). Our full coding sheet, including all of the obtained effect sizes, moderating variables, and $\mathrm{R}$ analysis scripts, can be found in the Supplemental Material available online.

\section{Search of studies}

Articles were retrieved through a computerized literature search of the electronic databases of the Education Resources Information Center (ERIC), PsychINFO, PubMed, and the Web of Science. A literature search was conducted for studies published up to November 2016 with three categories of key phrases used to search (a) key words containing variables concerning parenting or parent-child relationships (parent* or mother* or father* or parental or maternal* or attachment* or family* or bond*); (b) key words regarding self-control (self-control or self control or self-regulation or self regulation or self-discipline or self discipline or effortful-control or effortful control ${ }^{4}$; and (c) key words focusing on adolescents ${ }^{5}$ (adolescent* or adolescence or teen* or youth* or child* or student* or undergraduate or emerging adult* or young adult*).

\section{Inclusion criteria}

Studies were eligible for this meta-analysis when they met the following criteria. First, the study had to assess the relationship between any type of parenting (e.g., parental warmth, parental harshness) or the parentadolescent relationship (e.g., parental attachment, parental bond) and self-control. Specifically, the study had to report on self-control or interrelated concepts such as self-regulation, effortful control, or domainspecific forms of control such as impulse regulation. If no correlations were reported in the article, we contacted the corresponding author.

Second, the study had to focus on community-based samples, excluding clinical populations with psychological (e.g., cognitive impairments, autism) and/or physical (e.g., traumatic brain injury, diabetes, asthma) symptoms and/or criminal offenders. We are interested in the general population, and clinical groups may influence the magnitude or direction of effect sizes (Rothbaum \& Weisz, 1994).

Third, the mean age of the participants in the study had to fall within the age range of the adolescent period used in the current study (i.e., 10-22 years). The age range of adolescence is commonly considered to be between 12 and 18 years. However, we decided to broaden this range for two reasons. First, the beginning age of adolescence is related to puberty, and the World Health Organization (2017) has considered that the age of 10 years can be seen as the starting age of adolescence because of the earlier onset of puberty than in the past. Second, we consider the age of 22 years as the upper bound of adolescence because in this new era, youths have more time to develop instead of rushing to reproduce or make a living (National Research Council, 2015). In addition, a recent study suggests that 21 to 22 years can be seen as the cutoff age when adolescents become adults in terms of brain maturity (A. O. Cohen et al., 2016). In the case of longitudinal designs, the study had to assess at least one of the constructs (i.e., parenting or self-control) during adolescence. For example, in a longitudinal study that assessed parental warmth and self-control at the ages of 8,12 , and 14 years, the concurrent and longitudinal correlations within and between the ages of 12 and 14 years were included. Longitudinal correlations between the ages of 8 and 12 years and between 8 and 14 years were also included, but concurrent correlations at the age of 8 years were excluded.

Fourth, the study had to be published in English in a peer-reviewed journal, with the full text available for download. We did not include unpublished work, review articles, book chapters, dissertations, and conference abstracts, as findings in these forums are often subsequently published in peer-reviewed journals. The inclusion of only peer-reviewed articles has been widely accepted in prior meta-analyses (e.g., Karreman et al., 2006; Lovejoy, Graczyk, O'Hare, \& Neuman, 2000; Slagt et al., 2016). Moreover, research has shown that meta-analyses that include unpublished studies are just as likely to find evidence for publication bias as those that do not (Ferguson \& Brannick, 2012).

\section{Selection procedure}

The initial search in the databases yielded 6,792 hits after removing duplicates. The first two authors screened all abstracts independently, selecting articles for full-text reading. This resulted in 814 potentially relevant articles. These articles were carefully screened to determine whether they met the inclusion criteria. A number of articles were excluded because they did not include an appropriate measure of parenting and/or self-control $(k=252)$; the study consisted of a clinical population or a population in the wrong age range $(k=94)$; no full text in English was available $(k=76)$; the article was not published as a journal article $(k=$ 69); or no correlation table was available $(k=156)$. For the latter 156 articles, the corresponding authors were contacted by e-mail to request additional information. Some authors declined our invitation because they no longer had access to the data (6\%), some authors could not be contacted because no valid e-mail address was found $(12 \%)$, and others provided us with the necessary 
correlations (15\%, yielding 24 additional articles to include). Most of our e-mails remained unanswered (67\%). Finally, 191 studies met the selection criteria and were included in the meta-analysis. See Figure 1 for the PRISMA flowchart depicting the full search and inclusion process.

\section{Coding of the studies}

We developed a detailed coding scheme based on guidelines proposed by Lipsey and Wilson (2001), recording study descriptors and study characteristics potentially moderating the relation between parenting and self-control in adolescence. Study descriptors included basic information for all studies, such as author names, year of publication, article title, details on data collection, and sample size. Study characteristics possibly moderating the relation between parenting and self-control in adolescence were grouped into two moderator categories: moderators of theoretical interest and methodological characteristics.

\section{Theoretical moderators}

Type of parenting. To assess possible moderating effects of parenting types, we divided parenting practices and parenting characteristics into the three categories (Davis et al., 2017; Doyle \& Markiewicz, 2005; Gallarin \& Alonso-Arbiol, 2012; Hoeve et al., 2009; Karavasilis, Doyle, \& Markiewicz, 2003; Karreman et al., 2006; Slagt et al., 2016): positive parenting, including supervision, support, autonomy sensitivity, involvement, monitoring, authoritative parenting, warmth, and positive expression; negative parenting, including harsh parenting, neglect, rejection, negative expressions, authoritarian parenting, and permissiveness; and parent-child relationships, including social bonds, closeness, attachment, and security. Studies were coded as follows: $1=$ positive parenting, $2=$ negative parenting, or $3=$ relationship, respectively. See Table 1 for a detailed overview of the subdivision of parenting types.

Age. We coded age continuously. Some studies did not report age but school grade. If this was the case, we took the average age of that grade. For example, children in Grade 6 in the United States are on average between the ages of 11 and 12 years, and we therefore considered 11.5 years the mean age for this sample.

Culture. As mentioned above, we used the individualism index and ethnicity to represent culture. We coded the level of individualism of the country in which the data were collected according to Hofstede's individualism score (www.hofstede-insights.com). The score is a continuous index, with higher scores for more individualistic societies (e.g., an index of 91 for the United States) and lower scores for more collectivistic societies (e.g., an index of 17 for Taiwan). Regarding ethnicity, we coded the ethnicity of adolescents in the sample as follows: $1=$ balanced (i.e., no ethnicity exceeded $60 \%$ of the sample), $2=>60 \%$ White, $3=>60 \%$ African or African American, $4=>60 \%$ Asian or Asian American, $5=>60 \%$ Hispanic, or $6=$ other.

Adolescent gender. We coded adolescent gender categorically according to the percentage of boys and girls included in the sample as $1=$ overall balanced (the percentage of boys and girls in the sample ranged between $40 \%$ and $60 \%$ ), $2=>60 \%$ boys, or $3=>60 \%$ girls.

Parent gender. Studies were coded for whether the parenting referred specifically to adolescents' mothers or to adolescents' fathers. Often, however, parenting measures assessed parenting in general and did not specifically mention whether the measure focused on mothers, fathers, or both parents. As a result, we categorized the variable as follows: 1 = greater percentage of mothers (> $60 \%$ of the sample), $2=$ greater percentage of fathers ( $>60 \%$ of the sample), or $3=$ both parents, no clear percentage.

Multilevel analysis allows researchers to include multiple effect sizes from one study while simultaneously taking dependency into account. Thus, categories of positive and negative parenting and parent-child relationships were not mutually exclusive, with studies contributing effects sizes to multiple categories. Likewise, studies contributed to multiple ages if the design of the study was longitudinal and to parent gender if the effects of parenting on adolescent self-control were given separately for mothers and fathers. Nevertheless, the multilevel analyses allowed us to diminish the bias caused by the studies that provide multiple inputs.

\section{Methodological characteristics}

Report informant. Studies were coded for the informant of the parenting and self-control measures, respectively, as follows: 1 = adolescent self-report, $2=$ other-report (e.g., parent, mother, father), $3=$ observation, or $4=\mathrm{com}-$ posite (combining measures of multiple informants or assessment modalities).

Consistency report informant. To assess report informant consistency, we coded whether the parenting and self-control measures were assessed by the same informant as $1=$ consistent or $2=$ inconsistent. Attention was paid to the elements that made up the composite score. For example, if parenting and self-control were both 


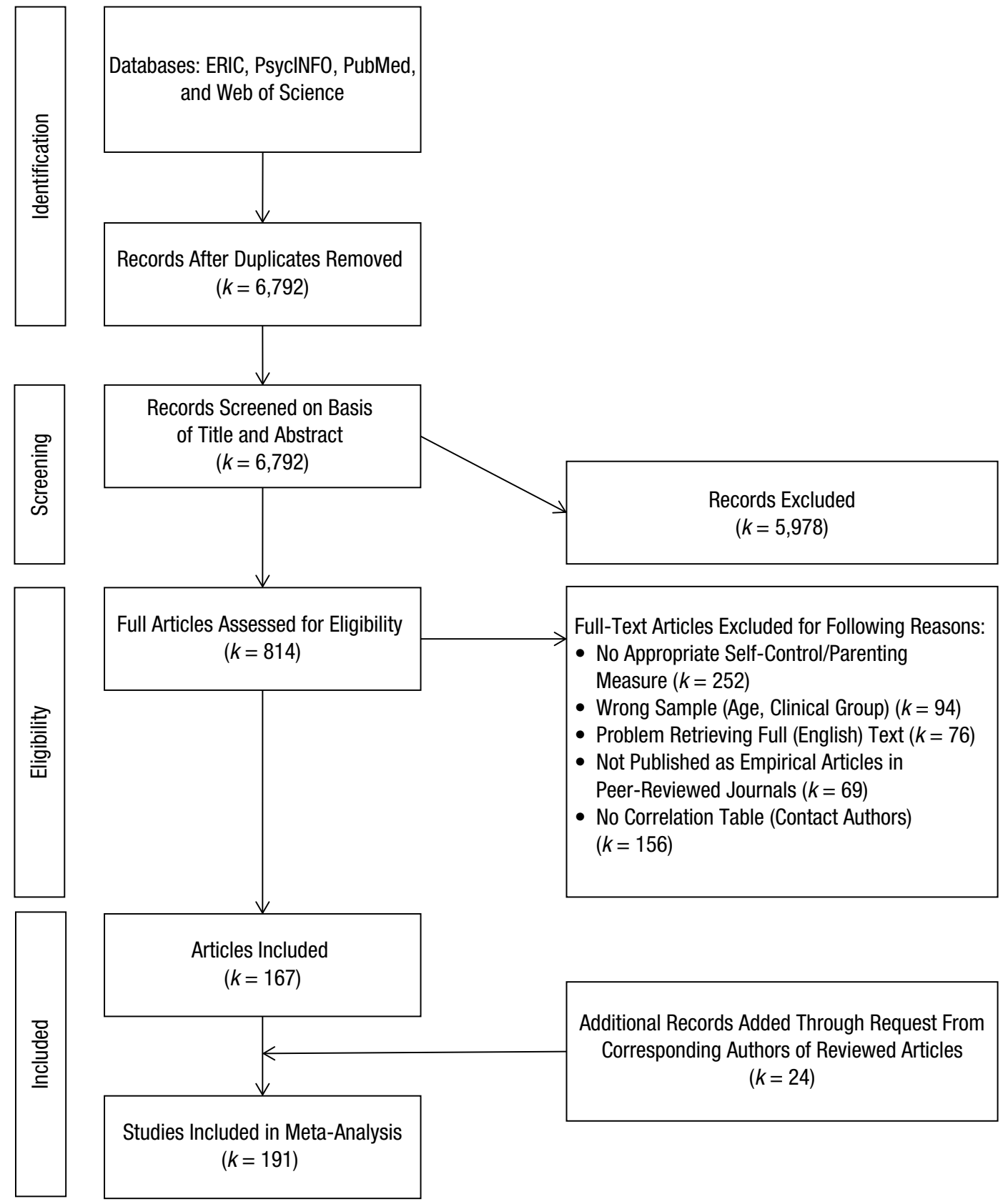

Fig. 1. Preferred Reporting Items for Systematic Reviews and Meta-Analyses (PRISMA) flowchart used to identify studies for detailed analysis of parenting and self-control. ERIC = Education Resources Information Center.

composite scores combined from the self-report and mother-report, then we considered the informant as consistent. If one composite score was combined from the self-report and mother-report whereas the other combined the self-report and teacher-report, we considered the informant as inconsistent.

Study design. Study design was coded as a categorical variable, including whether the effect size between parenting and self-control was derived from cross-sectional or longitudinal studies ( $1=$ cross-sectional, $2=$ longitudinal). For longitudinal studies we also included effect sizes for which parenting was measured first and selfcontrol was measured some time later (i.e., P $\rightarrow$ SC). Likewise, we included effect sizes for which self-control was measured first and parenting was measured some time later $(\mathrm{SC} \rightarrow \mathrm{P})$. As a result, we coded whether the effect size referred to the influence of parenting on selfcontrol or the influence of self-control on parenting as follows: $1=\mathrm{P} \rightarrow \mathrm{SC}$ or $2=\mathrm{SC} \rightarrow \mathrm{P}$. 


\section{Interrater agreement}

Of the 814 eligible articles, $20 \%$ were randomly selected to be double-coded by the first two authors. Intraclass correlation (for continuous variables) and Cohen's $\kappa$ (for categorical variables) were calculated. Intraclass correlations for continuous variables were high, ranging from .78 (for age) to 1.00 (for individualism score). Cohen's $\kappa$ for the categorical variables ranged from .91 (for including or excluding studies) to 1.00 (for study design, effect-size direction, and informant parenting). We resolved disagreement by in-depth reading and discussion based on the content of the article. Together, these results showed good interrater reliability, reflecting a good agreement for the study characteristics between the two independent raters.

\section{Effect sizes}

To investigate the magnitude of the relationship between parenting and self-control, we obtained Pearson's correlation coefficients obtained for all included studies (Lipsey \& Wilson, 2001). Zero-order correlation coefficients are bivariate estimates typically obtained from each empirical study's correlation matrix or requested from the authors if none was provided in the full text. To ensure a similar direction of effects, we recoded effect sizes if (a) parenting pertained to negative dimensions and (b) self-control was measured using a scale of lack of self-control or low self-control. We used Fisher's $r$-to- $z$ transformation (Fisher, 1921), converting the effect-size estimate from each association into an $E S_{Z}$ score to correct for skewness in the sampling distribution of $r$ (Lipsey \& Wilson, 2001). This $E S_{Z}$ score is assumed to approach normality, which is necessary for the accurate determination of mean effect-size estimates and for unbiased tests of statistical significance. As a result, $E S_{Z}$ scores were included in the analyses and transformed back to Pearson's $r$ for interpretation purposes (Field, 2001; Lipsey \& Wilson, 2001). ${ }^{6}$ For moderator analyses, categorical variable categories were transformed to $k-1$ dummy variables through binary coding (Assink \& Wibbelink, 2016; Lipsey \& Wilson, 2001).

\section{Publication bias}

Previous research has consistently shown that nonsignificant studies are more likely to be rejected for publication or remain unsubmitted by authors (Lipsey \& Wilson, 2001; Torgerson, 2006). This publication bias may result in inflated effect sizes and a restricted range of values in meta-analyses (Rosenthal, 1979). Therefore, it is important to statistically assess the possible influence of publication bias before interpreting the final results. In the current study, we handled this problem by applying a funnel plot, plotting the distribution of each individual study's effect size on the horizontal axis against its precision expressed in standard errors on the vertical axis (Torgerson, 2006). If a publication bias affects the data, an asymmetrical funnel plot is to be expected (Begg, 1994). In addition, Egger's test was applied to test the significance of the asymmetry of the plot, providing more precise information on the possible presence of publication bias (Egger, Smith, Schneider, \& Minder, 1997). When this test yielded significant results, sensitivity analyses were conducted by applying the trim-and-fill method, correcting for the asymmetric plots by imputing missing effect sizes through a number of iterations (Duval \& Tweedie, 2000a, 2000b). Imputing nonexisting effect sizes, however, is controversial, and effect sizes produced by the trim-and-fill analyses should be interpreted with caution (Sutton, Duval, Tweedie, Abrams, \& Jones, 2000).

\section{Data analyses}

All analyses were conducted in the metafor package (Viechtbauer, 2010) for the R software environment (Version 3.4.2; R Core Team, 2017). Most studies reported on multiple effect sizes. For example, some studies included longitudinal data (yielding effect sizes for different time points), different raters (resulting in effect sizes separately for the mother-report and fatherreport), and effect sizes separately for boys and girls. It is likely that these effect sizes from the same study are more similar than effect sizes from different studies because they rely on the same sample, data collection, and sampling methods. When using nested effect sizes, however, the assumption of traditional meta-analyses that observations are independent and error terms are uncorrelated is violated (Lipsey \& Wilson, 2001; Rosenthal, 1984). Not taking into account this dependency can result in a biased result, as it may create artificially narrow confidence intervals and shrunken standard errors favoring statistical significance (Hox, Moerbeek, \& van de Schoot, 2010).

Strategies applied to handle this dependency problem include selecting one effect size from each study, averaging effect sizes within studies, or simply ignoring the dependency of effect sizes (Lipsey \& Wilson, 2001). However, more recently the multilevel meta-analysis has been suggested as a more preferable tool, as it takes into account dependency while including all available effect sizes, resulting in maximum information and greater statistical power (Assink \& Wibbelink, 2016; Hendriks et al., 2017; Hox et al., 2010; Van den Noortgate et al., 2013). Three-level models apply when 
groups are nested within clusters and thus are not independent from one another. In our case, we have variance at the effect-size level (Level 1) that is nested within a sample (Level 2; e.g., effect sizes based on the National Longitudinal Study of Adolescent to Adult Health, or Add Health, data set; Harris et al., 2009), with variance also between studies (Level 3; taking into account that effect sizes vary between studies). Incorrectly modeling this dependency in the data will likely result in biased standard errors, leading to incorrect inferences about the relationships being studied (Viechtbauer, 2010).

Accordingly, we applied a three-level model to account for the three sources of variance: Level 1 takes into account the sampling variance of the effect sizes; Level 2 takes into account the variance between effect sizes from the same sample, allowing effect sizes to vary within studies; and Level 3 takes into account the variance between studies, allowing effect sizes to vary between studies (Hox et al., 2010; Van den Noortgate et al., 2013). In addition, because parameter estimates from different levels of analyses are not independent in this multilevel approach, no greater weight will be placed on studies with more effect sizes. Thus, a study that, for example, includes 10 effect sizes will not contribute 10 times more to the mean effect size than a study that has only one effect size (Van den Noortgate et al., 2013). Overall, multilevel modeling allows including effect sizes on the basis of the same sample, providing more precision in estimating mean effect sizes while simultaneously modeling the nestedness of the data (Cheung, 2014; Van den Noortgate et al., 2013).

The current three-level analysis was conducted in three stages. First, the overall mean effect sizes were estimated to assess the strength of the association between parenting and self-control in adolescence. Second, we applied a likelihood-ratio test to assess between-study and within-study heterogeneity. It is important to note that the level of "study" entails the data set. For example, multiple papers are based on the Add Health data set. Accordingly, for the multilevel analyses, we gave studies using the same data set (e.g., all published studies using Add Health data) the same study ID, clustering them as if they were all from one published study. This allowed us to take into account this dependency, referring to the included studies as the number of independent studies.

Third, if there was evidence for heterogeneity in effect sizes (presented as a $Q_{E}$ that, when significant, indicated heterogeneity among effect sizes), moderation analyses were conducted for moderators of theoretical interest and methodological characteristics. To obtain reliable results, we conducted moderator analyses only if each category contained at least five studies (parameter estimates are poor when the number of studies is very small; Weisz et al., 2017).

The three-level analyses were conducted according to the three-level random-effects model guidelines formulated by Assink and Wibbelink (2016). The analyses used the restricted maximum-likelihood procedure for parameter estimation and were performed with the metafor package for R. Moreover, we used G*Power (Version 3.1.9.2; Faul, Erdfelder, Lang, \& Buchner, 2007) to calculate the sample size needed for future research to obtain the mean correlation found in this research. Sample sizes for obtaining four levels of power (i.e., $.80, .90, .95$, and .99) with an alpha level of .05 were recommended.

\section{Results}

\section{Descriptive statistics}

The current meta-analysis included 191 articles reporting on 159 independent studies and comprising 1,540 effect sizes. The overall sample size ${ }^{7}$ was $N=164,459$, with study sample sizes ranging from $N=47$ (Samuelson, Krueger, \& Wilson, 2012) to $N=19,810$ (Barnes \& Morris, 2012), and a mean age of 13.92 years. The publication year of the included studies ranged from 1990 to 2016; the number of studies published annually is given in Figure 2. Most studies included both boys and girls (80\%); some studies focused specifically on boys (7\%), some focused specifically on girls (9\%), and some studies did not specify gender (4\%). Some studies focused on parenting in general without differentiating between mothers and fathers (44\%), some specifically focused on maternal parenting (40\%), and a few studies specifically focused on paternal parenting (16\%). Of all included effect sizes, 53\% focused on positive parenting, 33\% focused on negative parenting, and 14\% focused on parent-child relationships (for details on parenting dimensions, see Table 1).

Table 1 presents the details on parenting categories. It shows that positive parenting predominantly comprises authoritative parenting, monitoring, and support, whereas negative parenting comprises conflict, authoritarian parenting, psychological negative control, harsh parenting, and, to a lesser degree, coercion and withdrawal. Finally, parent-child relationships mostly comprise attachment, whereas alienation and trust from the attachment scale make up the smallest percentage of this category.

Studies were conducted worldwide, including in Australia, Canada, China, India, and Mexico, with most effect sizes retrieved from the United States (62\%), South Korea (10\%), Switzerland (6\%), and the Netherlands (4\%; for a graphical representation of the countries represented in the current meta-analysis, see Fig. 3). Hofstede's 


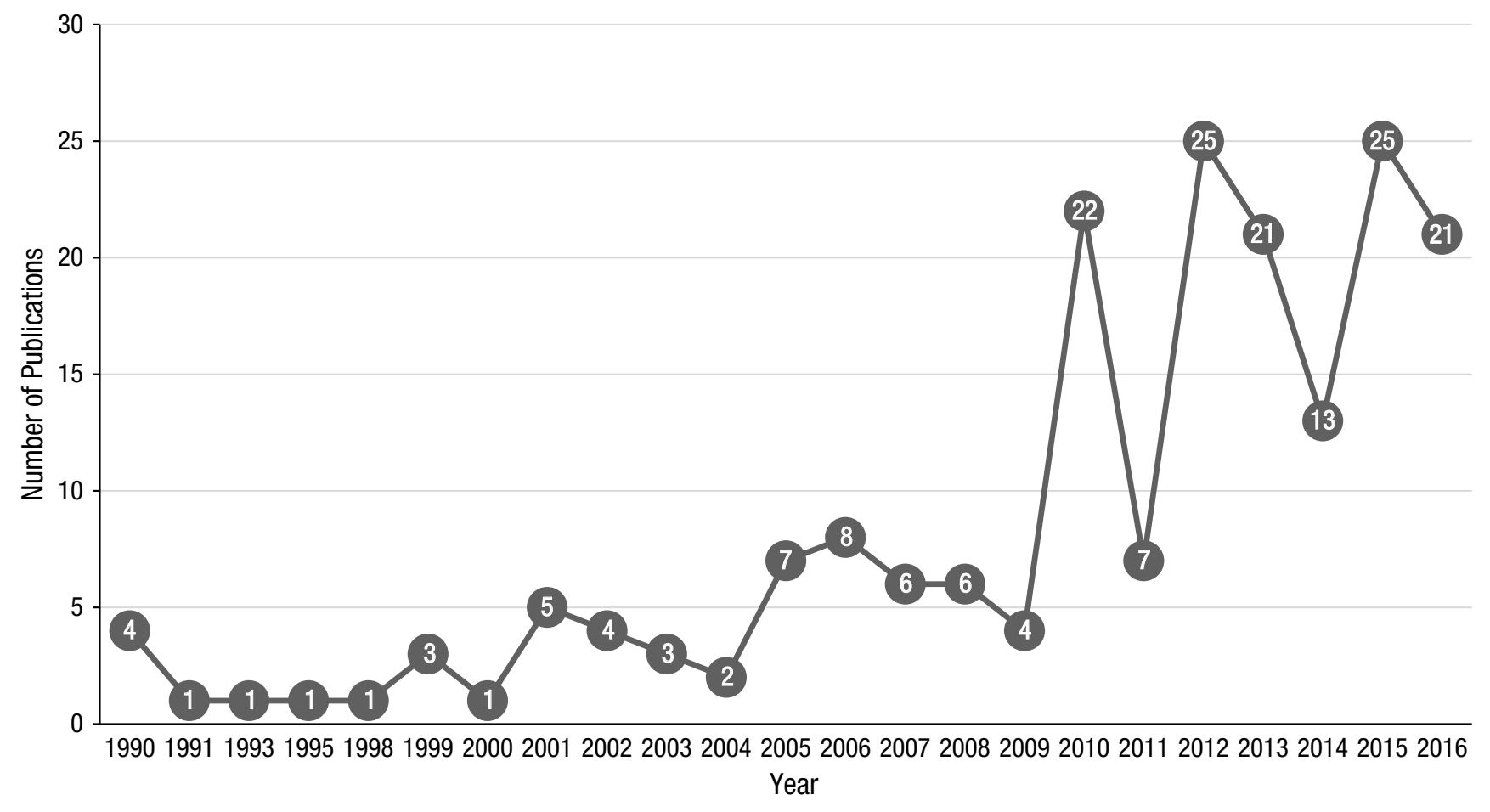

Fig. 2. Number of included studies published annually.

individualism index ranged between 17 (Taiwan)/18 (South Korea) and 90 (Australia)/91 (United States), with $k=28$ independent studies reporting on countries with an individualism score $<50$ and $k=129$ studies reporting on countries with an individualism score $>50$.

The current meta-analysis included both crosssectional (57\%) and longitudinal (43\%) effect sizes. Of the longitudinal effects, 56\% measured parenting first and self-control some time later ( $\rightarrow$ SC), and 44\% measured self-control first and parenting some time later (SC $\rightarrow$ P). Longitudinal studies ranged from 1 month to 13 years; most studies reported on a 0 - to 1 -year time lag $(57 \%)$ or a 1 - to 2 -year time lag (23\%), and others reported on a 2- to 3-year time lag (5\%), 3- to 4-year time lag (13\%), 4- to 5-year time lag (2\%), and > 5-year time lag (16\%). Thus, the current meta-analysis included (a) cross-sectional effect sizes; (b) longitudinal P $\rightarrow$ SC effect sizes, where parenting predicted subsequent selfcontrol; and (c) longitudinal SC $\rightarrow$ P effect sizes, where adolescent self-control predicted subsequent parenting. These three groups describe different patterns of effects and should be treated individually. Therefore, we assessed the overall effects of these three groups separately, followed by statistical analyses testing whether these effects statistically differed. Of the 1,540 effect sizes, 876 concerned cross-sectional associations, 373 concerned longitudinal P $\rightarrow$ SC associations, and 291 concerned longitudinal SC $\rightarrow$ P associations.

\section{Overall effects}

Cross-sectional association. The overall effect size of cross-sectional studies was statistically significant, $E S_{Z}=$ $0.207, S E=0.010, t=20.165, p<.001,95 \%$ confidence interval $(\mathrm{CI})=[0.187,0.227]$, with substantial heterogeneity, $Q_{E}(875)=13,140.584, p<.001$. Using an inverse version of Fisher's $r$-to- $z$ formula, we transformed this effect size back to Pearson's $r$ for interpretation purposes. We found that the cross-sectional association between parenting and self-control in adolescence was $r=.204,95 \%$ $\mathrm{CI}=[.185, .223]$.

Longitudinal associations: $\boldsymbol{P} \rightarrow \boldsymbol{S C}$. The overall effect size of longitudinal $\mathrm{P} \rightarrow \mathrm{SC}$ was also statistically significant, $E S_{Z}=0.158, S E=0.015, t=10.238, p<.001,95 \%$ $\mathrm{CI}=[0.128,0.188]$, with substantial heterogeneity, $Q_{E}(372)=3,349.047, p<.001$. We found that the longitudinal association for which parenting was measured first and self-control later was $r=.157,95 \% \mathrm{CI}=[.127, .186]$.

Longitudinal associations: $\boldsymbol{S C} \rightarrow \boldsymbol{P}$. For longitudinal $\mathrm{SC} \rightarrow \mathrm{P}$, the overall effect size was also statistically significant, $E S_{Z}=0.156, S E=0.022, t=7.123, p<.001,95 \%$ $\mathrm{CI}=[0.113,0.199]$, with substantial heterogeneity, $Q_{E}(290)=$ $2,293.718, p<.001$. The longitudinal association for which self-control was measured first and parenting later was $r=.155,95 \% \mathrm{CI}=[.113, .196]$. 


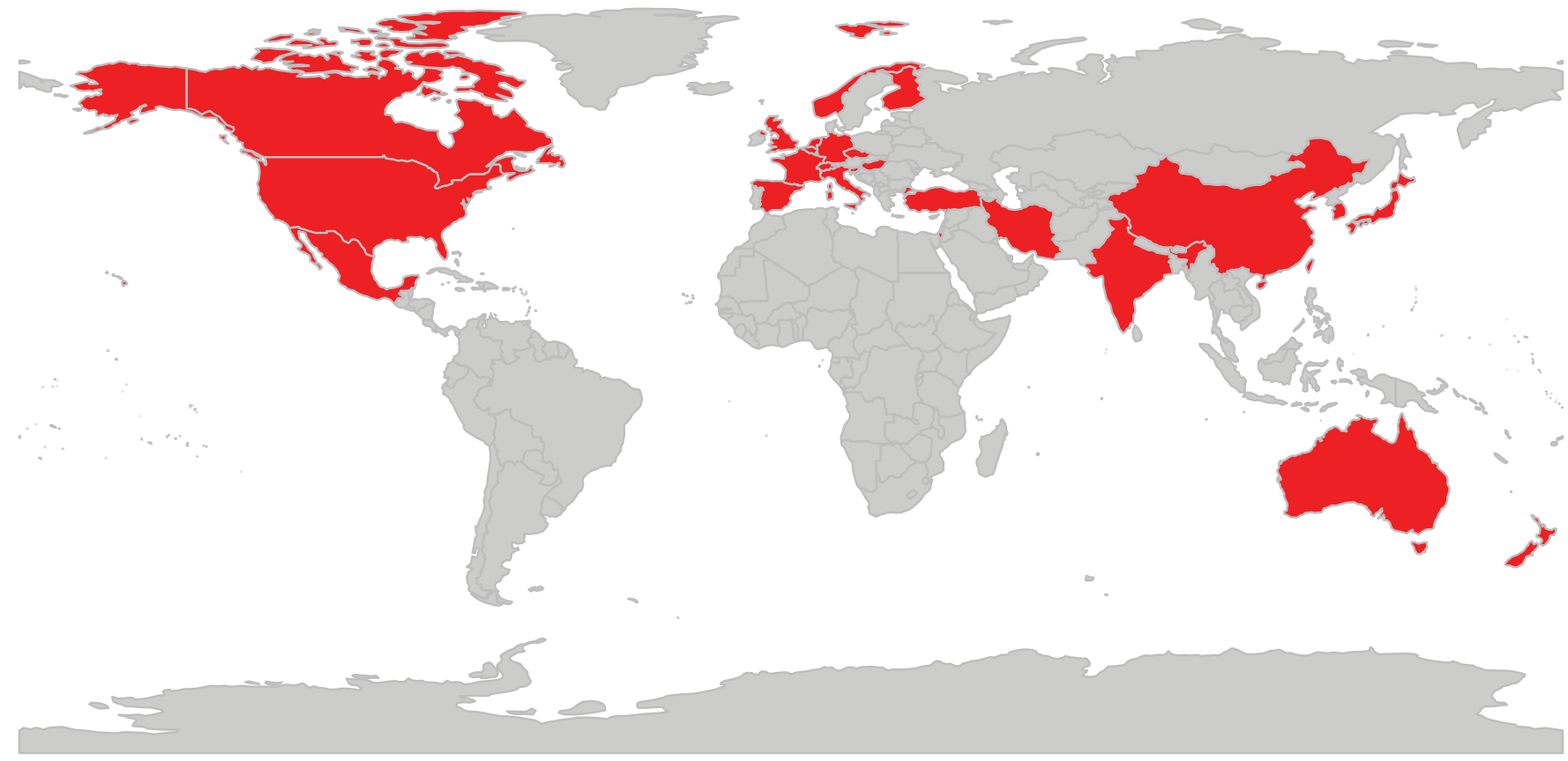

Fig. 3. Graphical representation of countries represented in the meta-analysis.

Comparison between cross-sectional and longitudinal effect sizes. As reported above, there were some observed differences in the effect sizes between crosssectional and longitudinal studies, and therefore we tested whether this difference was statistically significant. Applying three-level analyses, we found an overall significant difference between the three groups, $F(2,1537)=26.136$, $p<.001$, and significant heterogeneity in effect sizes, $Q_{E}(1537)=18,783.349, p<.001$. As shown in Table 2, the associations for longitudinal $\mathrm{P} \rightarrow$ SC were significantly smaller than cross-sectional associations. Likewise, associations for longitudinal SC $\rightarrow$ P were also significantly smaller than cross-sectional associations. Results showed no significant difference in the associations between longitudinal $\mathrm{P} \rightarrow \mathrm{SC}$ and $\mathrm{SC} \rightarrow \mathrm{P}$.

Analyses for these three groups were then conducted separately. First, a random-effects model was used to calculate variance at the sampling, within-study, and between-study levels. Second, multiple potential moderators were tested individually. Third, a multiplemoderator model including all the significant moderators was performed to control for the associations among moderators. Finally, we also checked publication bias using the funnel plot (Egger et al., 1997).

\section{Cross-sectional studies}

Variance of the overall effect size. The variance at the within-study level (estimate $=.010, p<.001$ ) and the between-study level (estimate $=.011, p<.001$ ) were both significant. Follow-up analyses found that variance at the sampling, within-study, and between-study levels was $5.13 \%, 45.11 \%$, and $49.76 \%$, respectively. Hunter and Schmidt (1990) proposed that heterogeneity can be considered as substantial if less than $75 \%$ of the variance can be attributed to the sampling variance and that in this case examination of the moderating effects of the study and/or effect-size characteristics on the overall effect can be fruitful. In our study, only $5.13 \%$ of the variance was explained by the samples, indicating that the continuous exploration of potential moderators was meaningful.

Moderator analyses. Considering the large statistical power, we were confident in assessing potential moderators of theoretical and methodological interest (see Table 3). Regarding the moderators of theoretical interest, the only significant moderator was type of parenting, $Q_{E}(873)=12,763.277, p<.001, F(2,873)=3.483, p=.031$. None of the other moderators of theoretical interest, including cultural characteristics (ethnicity, Hofstede's individualism), parent gender (i.e., whether the effect refers to mother or father), adolescent gender (i.e., whether the effect refers to boys and girls), or age of adolescents, ${ }^{8}$ was significant.

Pertaining to the moderators of methodological interest, all three moderators in this category were significant: report informant of parenting measure, $Q_{E}(871)=$ $13,034.230, p<.001, F(3,871)=5.172, p=.002$, report informant of self-control measure, $Q_{E}(872)=12,859.772$, $p<.001, F(3,872)=5.068, p=.002$, and consistency 
Table 2. Comparison of Cross-Sectional and Longitudinal Effect Sizes

\begin{tabular}{|c|c|c|c|c|c|}
\hline Pairwise comparison & $E S_{z}$ & $S E$ & $t$ & $95 \% \mathrm{CI}$ & $p$ \\
\hline$\Delta$ Slope cross-sectional (vs. P $\rightarrow$ SC) & -0.050 & 0.008 & -6.228 & {$[-0.066,-0.034]$} & $<.001$ \\
\hline$\Delta$ Slope cross-sectional (vs. SC $\rightarrow$ P) & -0.052 & 0.009 & -6.058 & {$[-0.068,-0.035]$} & $<.001$ \\
\hline$\Delta$ Slope $\mathrm{P} \rightarrow \mathrm{SC}($ vs. SC $\rightarrow \mathrm{P})$ & -0.002 & 0.009 & -0.174 & {$[-0.019,0.016]$} & .862 \\
\hline$Q_{E}(d f)$ & \multicolumn{5}{|c|}{$18,783.349(1537), p<.001$} \\
\hline Omnibus test & \multicolumn{5}{|c|}{$F(2,1537)=26.136, p<.001$} \\
\hline Variance within study & \multicolumn{5}{|c|}{$.008, p<.001$} \\
\hline Variance between studies & \multicolumn{5}{|c|}{$.010, p<.001$} \\
\hline Number of ESs & \multicolumn{5}{|c|}{1,540} \\
\hline
\end{tabular}

Note: $\mathrm{CI}=$ confidence interval; $\mathrm{ES}=$ effect size; $\mathrm{P}=$ parenting; $\mathrm{SC}=$ self-control.

of the report informant of the parenting and self-control measures, $Q_{E}(873)=13,104.212, p<.001, F(1,874)=$ $15.043, p<.001$.

Significant moderators. A follow-up comparison based on the significant moderators found above was conducted, and the results are summarized in Table 4. Regarding the type of parenting, we found that the effect sizes for the associations betweeen positive parenting and selfcontrol, between negative parenting and self-control, and between parent-child relationship and self-control were all significant. Results of further comparison suggested that the relationship for negative parenting was significantly smaller than that for positive parenting.

Regarding the informant of parenting measure, we found that the effect sizes of the relationship between parenting and self-control were all significant when parenting was measured using adolescent self-report, other-report, observation, and composite measures. Results of a further comparison indicated that effect sizes for studies using composite measures were significantly larger than those using self-report, otherreport, and observation and that effect sizes for studies using observation measures to assess parenting were significantly lower than those using adolescent selfreport and other-report.

With respect to the informant of self-control measures, we found that effect sizes of the relationship between parenting and self-control were all significant when self-control was assessed using adolescent selfreport, other-report, observation, and composite measures. A follow-up comparison indicated that effect sizes of studies using composite measures to assess self-control were significantly larger than those using adolescent self-report, other-report, and observation and that effect sizes of studies using observation measures to assess self-control were significantly lower than those using adolescent self-report.

For the consistency of the report informant on parenting and self-control measures, effect sizes were both significant for studies using consistent and inconsistent report informants. Results of a follow-up comparison showed that effect sizes of studies using inconsistent report informants were significantly lower than those using consistent report informants.

Multiple-moderator model. According to Hox et al. (2010), moderators may be interrelated, possibly causing

Table 3. Cross-Sectional Associations: The $Q_{E}$ Statistics Testing Residual Heterogeneity and the Omnibus to Test the Effect of the Moderators

\begin{tabular}{lrrrr}
\hline Moderator & \multicolumn{1}{c}{$Q_{E}(d f)$} & $p$ & Omnibus test & $p$ \\
\hline Theoretical moderators & & & & \\
$\quad$ & & & \\
$\quad$ Age & $10,420.319(830)$ & $<.001$ & $F(1,830)=1.632$ & .202 \\
$\quad$ Ethnicity of adolescents & $9,503.564(722)$ & $<.001$ & $F(5,722)=1.286$ & .268 \\
$\quad$ Hofstede's individualism & $12,883.360(862)$ & $<.001$ & $F(1,862)=0.300$ & .584 \\
$\quad$ Type of parenting & $12,763.277(873)$ & $<.001$ & $F(2,873)=3.483$ & .031 \\
$\quad$ Parent gender & $13,035.444(865)$ & $<.001$ & $F(2,865)=2.743$ & .065 \\
$\quad$ Adolescent gender & $10,777.295(840)$ & $<.001$ & $F(2,840)=1.443$ & .237 \\
Methodological moderators & & & & \\
$\quad$ Report informant of parenting measure & $13,034.230(871)$ & $<.001$ & $F(3,871)=5.172$ & .002 \\
$\quad$ Report informant of self-control measure & $12,859.772(872)$ & $<.001$ & $F(3,872)=5.068$ & .002 \\
$\quad$ Consistency of informants & $13,104.212(874)$ & $<.001$ & $F(1,874)=15.043$ & $<.001$ \\
\hline
\end{tabular}




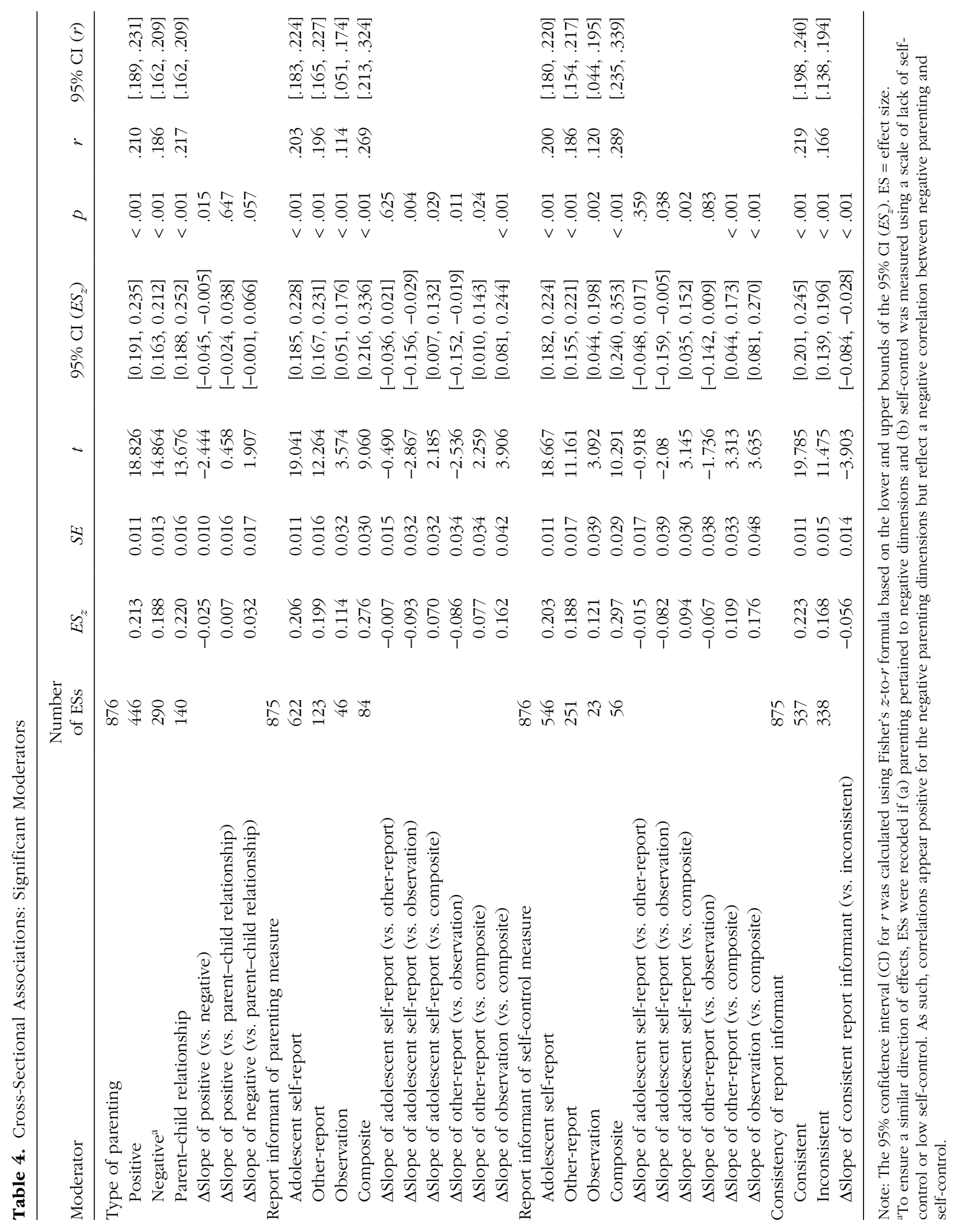


Table 5. Cross-Sectional Association: Results for the Multiple Moderator Model

\begin{tabular}{|c|c|c|c|c|}
\hline Moderator variables & $\beta(S E)$ & $95 \% \mathrm{CI}$ & $t$ & $p$ \\
\hline Type of parenting: negative parenting (vs. positive parenting) & $-0.028(0.010)$ & {$[-0.048,-0.007]$} & -2.675 & .008 \\
\hline Report informant of parenting: other-report (vs. self-report) & $-0.008(0.015)$ & {$[-0.037,0.021]$} & -0.557 & .578 \\
\hline Report informant of parenting: observation (vs. self-report) & $-0.058(0.034)$ & {$[-0.125,0.009]$} & -1.713 & .087 \\
\hline Report informant of parenting: composite (vs. self-report) & $0.050(0.034)$ & {$[-0.017,0.117]$} & 1.465 & .143 \\
\hline Report informant of self-control: observation (vs. self-report) & $-0.079(0.041)$ & {$[-0.158,0.001]$} & -1.936 & .053 \\
\hline Report informant of self-control: composite (vs. self-report) & $0.126(0.033)$ & {$[0.061,0.191]$} & 3.829 & $<.001$ \\
\hline Consistency of report informant: inconsistent (vs. consistent) & $-0.069(0.016)$ & {$[-0.099,-0.038]$} & -4.422 & $<.001$ \\
\hline Omnibus test & \multicolumn{4}{|c|}{$F(9,865)=6.157, p<.001$} \\
\hline Variance within study & \multicolumn{4}{|c|}{$.009, p<.001$} \\
\hline Variance between studies & \multicolumn{4}{|c|}{$.011, p<.001$} \\
\hline
\end{tabular}

Note: $\mathrm{CI}=$ confidence interval; $\mathrm{ES}=$ effect size

multicollinearity problems in the analyses. To overcome these problems, we constructed a multiple-moderator model that included all significant moderators found in the individual moderation test above. The results of this model are summarized in Table 5. An omnibus test showed significant results, $F(9,865)=6.157, p<.001$, suggesting that at least one of the regression coefficients of the moderators significantly deviated from zero. These results indicated that negative parenting (vs. positive parenting), composite measures of self-control (vs. adolescent self-reports), and inconsistent report informants (vs. consistent report informants) had unique moderating effects on the relationship between parenting and self-control.

Publication bias. Considering our large sample size, and that, for numerous studies, the association between parenting and self-control was not the primary research interest, we assumed little influence of publication bias. To statistically check this assumption, we inspected funnel plots using Fisher's $z$ transformations (see Figs. 4-6) and applied Egger's regression test (Egger et al., 1997; Torgerson, 2006). Results of the regression test for funnelplot asymmetry showed that there was no significant asymmetry $(z=-1.506, p=.132)$, suggesting that no significant publication bias was detected for the results found above.

\section{Longitudinal $P \rightarrow$ SC studies}

Variance of the overall effect size. The variance at the within-study level (estimate $=.006, p<.001$ ) and between-study level (estimate $=.007, p<.001$ ) were both significant. Follow-up analyses found that variance at the sampling, within-study, and between-study levels was
$7.01 \%, 44.29 \%$, and $48.69 \%$, respectively, indicating that the continuous exploration of potential moderators was meaningful.

Moderator analyses. Similar to cross-sectional analyses, moderators of theoretical and methodological interest were tested in the longitudinal data and are summarized in Table 6. Moderator analyses were conducted only if each category contained at least five studies (parameter estimates are poor when the number of studies is very small). As a result, we could not test the moderating effect of adolescent gender and report informant of self-control. Regarding the moderators of theoretical interest, the only significant moderator was parent gender, $Q_{E}(366)=3,188.953, p<.001, F(2,366)=6.150$, $p=.002$. None of the other moderators of theoretical interest, including type of parenting (positive, negative, relationship) or cultural characteristics (ethnicity, Hofstede's individualism), was significant.

To analyze the moderators of methodological interest, we tested informants of parenting measures and the consistency of informants. Both report informants of parenting measures, $Q_{E}(360)=3,132.167, p<.001$, $F(3,360)=3.770, p=.011$, and the consistency of informants, $Q_{E}(371)=3,186.385, F(1,371)=6.562, p=$ .011, yielded significant results.

Significant moderators. Follow-up analyses were conducted to analyze the aforementioned significant moderators. The results are presented in Table 7. Regarding parent gender, we found significant associations for maternal parenting-self-control, paternal parenting-self-control, and both parents' parenting-self-control. Results of further comparison suggested that the association was 


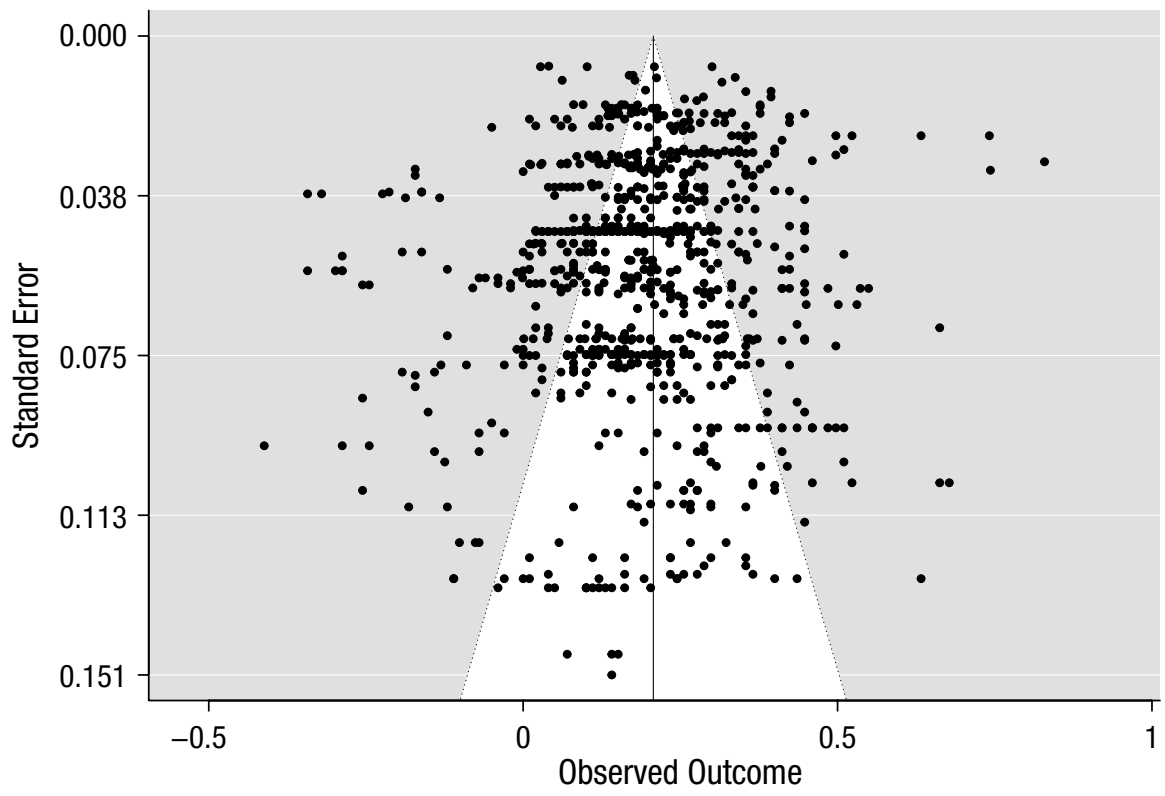

Fig. 4. Funnel plot for the associations between parenting and self-control in cross-sectional studies.

significantly smaller for both parents' parenting than for either maternal or paternal parenting. The association between parenting and self-control was not significantly different for mothers and fathers.

With respect to the report informant of parenting measures, we found that effect sizes of the relationship between parenting and self-control were all significant when parenting was assessed using adolescent self-report, other-report, observation, and composite measures. A follow-up comparison indicated that effect sizes of studies using composite measures to assess parenting were significantly larger than those using self-report, other-report, and observation.

For the consistency of the report informant of parenting and self-control measures, effect sizes were both significant for studies using consistent and inconsistent

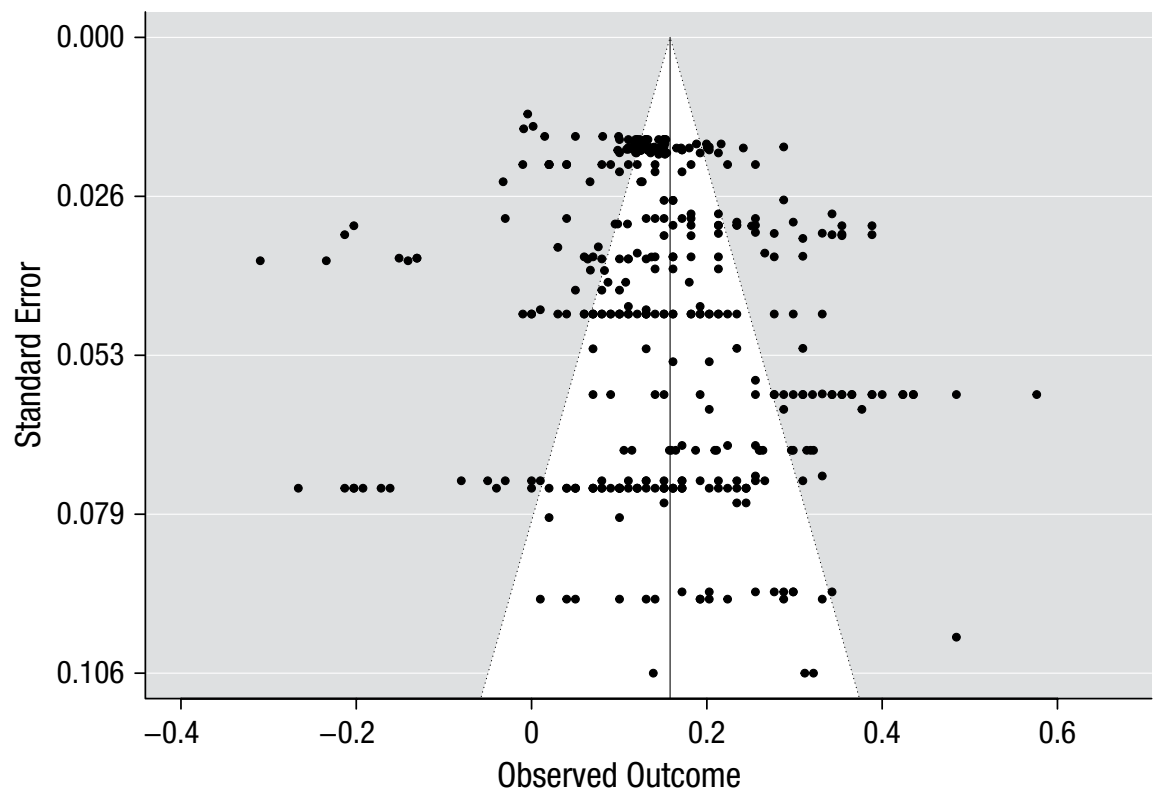

Fig. 5. Funnel plot for the associations between parenting and self-control for longitudinal studies in which parenting was measured first and self-control was measured some time later. 


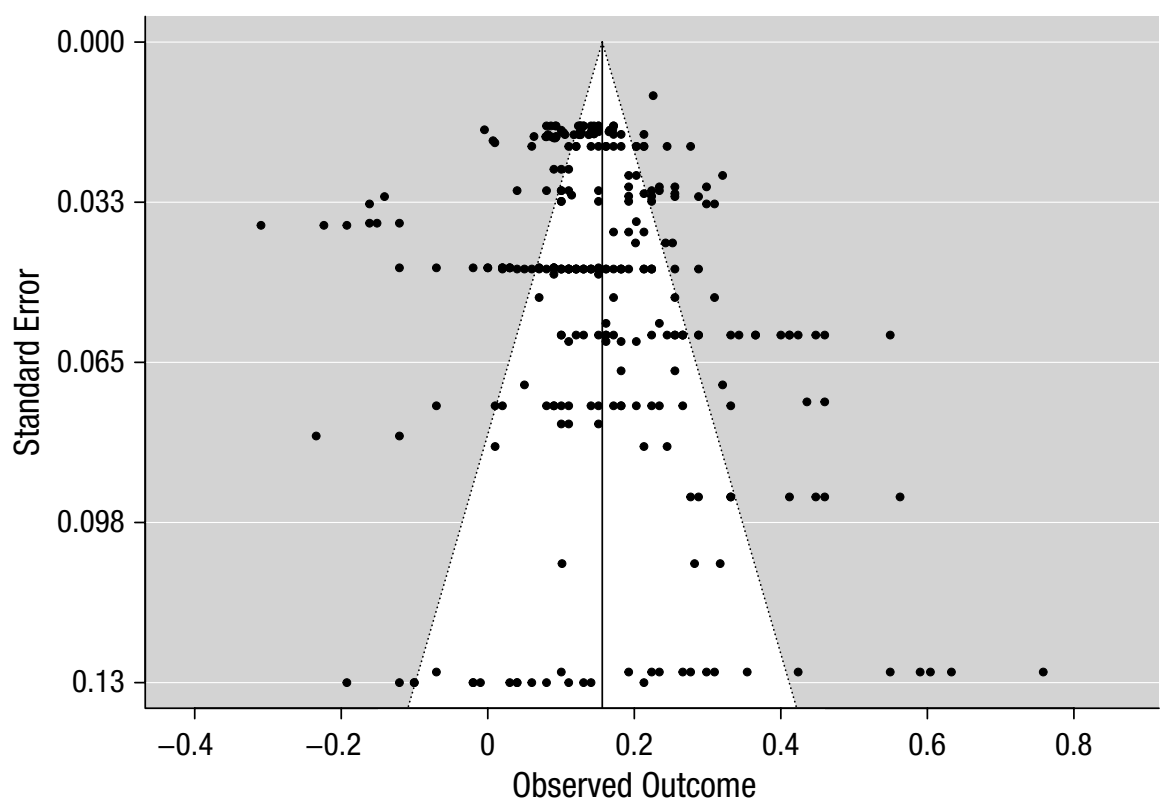

Fig. 6. Funnel plots for the associations between parenting and self-control for longitudinal studies in which self-control was measured first and parenting was measured some time later.

report informants. Results of a follow-up comparison showed that effect sizes of studies using inconsistent report informants were significantly larger than those using consistent report informants.

Multiple-moderator model. For the multiple-moderator model, all significant moderators illustrated in Table 8 were included into one single model to test their robustness. The results suggested that at least one of the regression coefficients of the moderators significantly deviated from zero, $F(6,353)=4.278, p<.001$. These results indicated that parent gender (both vs. mother) and informant reports (inconsistent vs. consistent) had unique moderating effects on the relationship between parenting and self-control.
Publication bias. The funnel plot is illustrated in Figure 5. The results of the regression test suggest significant asymmetry $(z=2.389, p=.017)$, which indicates that there is publication bias for longitudinal studies on the association between parenting and self-control. A trimand-fill procedure was applied to take publication bias into account, resulting in an adjusted effect size of $E S_{z}=$ $0.106, S E=0.007,95 \% \mathrm{CI}=[0.092,0.119], p<.001, r=$ $.105,95 \% \mathrm{CI}=[.092, .118]$.

\section{Longitudinal $S C \rightarrow P$}

Variance of the overall effect size. The variance at the within-study level (estimate $=.005, p<.001$ ) and the between-study level (estimate $=.012, p<.001$ ) were both

Table 6. Longitudinal Associations for Parenting Measured First and Self-Control Measured Later: The $Q_{E}$ Statistics Testing Residual Heterogeneity and the Omnibus to Test the Effects of the Moderators

\begin{tabular}{lcccc}
\hline Moderator & $Q_{E}(d f)$ & $p$ & Omnibus test & $p$ \\
\hline Theoretical moderators & & & & \\
$\quad$ & & & & \\
$\quad$ Ethnicity of adolescents & $2,623.127(319)$ & $<.001$ & $F(5,319)=0.233$ & .948 \\
$\quad$ Hofstede's individualism & $3,345.279(371)$ & $<.001$ & $F(1,371)=0.422$ & .516 \\
$\quad$ Type of parenting & $3,243.333(370)$ & $<.001$ & $F(2,370)=1.303$ & .273 \\
$\quad$ Parent gender & $3,188.953(366)$ & $<.001$ & $F(2,366)=6.150$ & .002 \\
Methodological moderators & & & & \\
$\quad$ Report informant of parenting measure & $3,132.167(360)$ & $<.001$ & $F(3,360)=3.770$ & .011 \\
$\quad$ Consistency of informants & $3,186.385(371)$ & $<.001$ & $F(1,371)=6.562$ & .011 \\
\hline
\end{tabular}




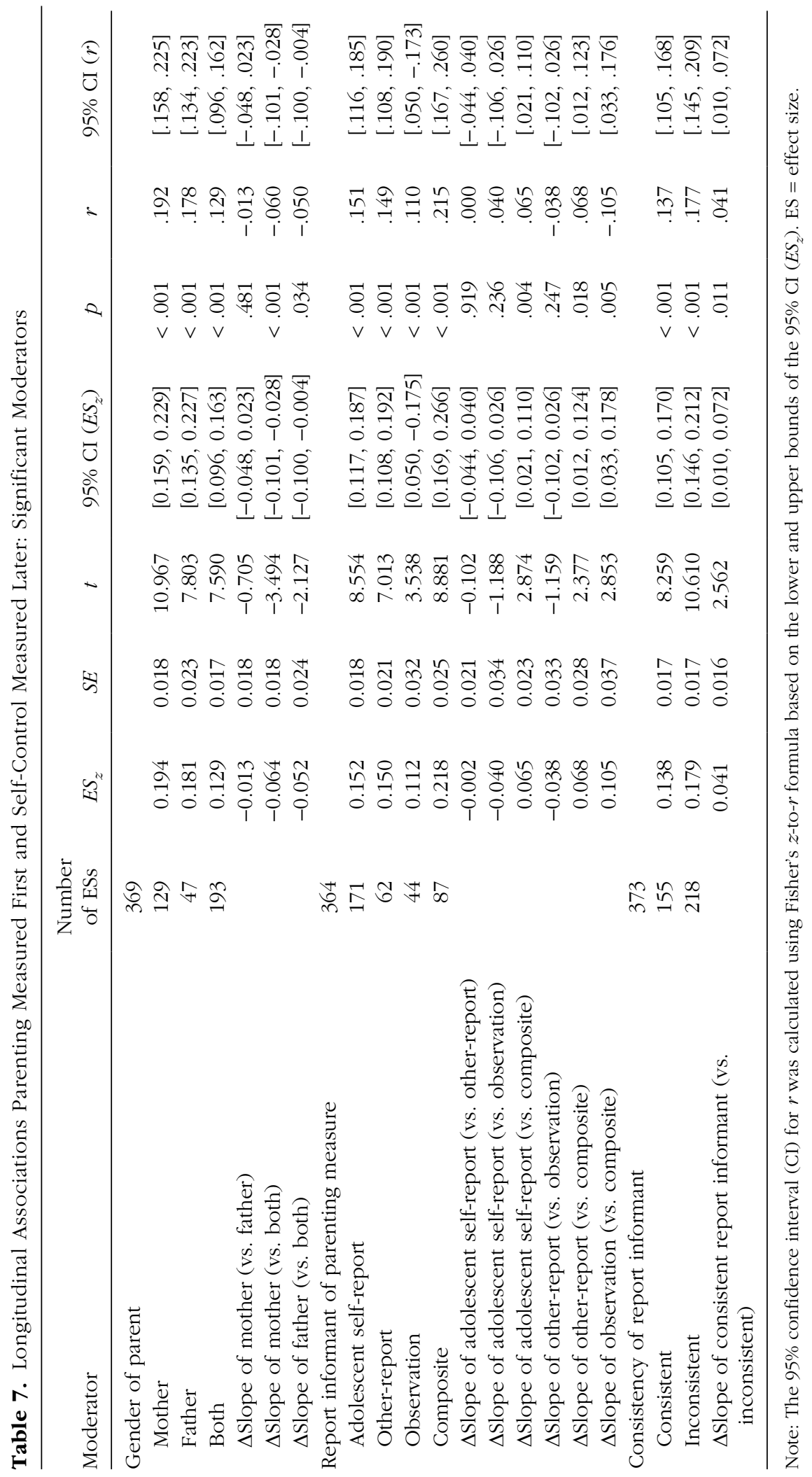


Table 8. Longitudinal Association for Parenting Measured First and Self-Control Measured Late: Results for the Multiple Moderator Model

\begin{tabular}{|c|c|c|c|c|}
\hline Moderator variables & $\beta(S E)$ & $95 \% \mathrm{CI}$ & $t$ & $p$ \\
\hline Gender parent: father (vs. mother) & $-0.016(0.018)$ & {$[-0.051,0.019]$} & -0.900 & .369 \\
\hline Report informant of parenting measure: other report (vs. self-report) & $-0.022(0.023)$ & {$[-0.067,0.022]$} & -0.985 & .325 \\
\hline Report informant of parenting measure: observation (vs. self-report) & $-0.067(0.037)$ & {$[-0.0140,0.005]$} & -1.835 & .067 \\
\hline Report informant of parenting measure: composite (vs. self-report) & $0.030(0.026)$ & {$[-0.021,0.081]$} & 1.461 & .253 \\
\hline Omnibus test & \multicolumn{4}{|c|}{$F(6,353)=4.278, p<.001$} \\
\hline Variance within study & \multicolumn{4}{|c|}{$.006, p<.001$} \\
\hline Variance between studies & \multicolumn{4}{|c|}{$.005, p<.001$} \\
\hline Number of ESs & \multicolumn{4}{|c|}{360} \\
\hline
\end{tabular}

Note: $\mathrm{CI}=$ confidence interval; $\mathrm{ES}=$ effect size

significant. Follow-up analyses found that variance at the sampling, within-study, and between-study levels was $5.46 \%, 26.29 \%$, and $68.26 \%$, respectively, indicating that the continuous exploration of potential moderators was meaningful.

Moderator analyses. Similar to longitudinal $\mathrm{P} \rightarrow \mathrm{SC}$ analyses, the moderating effect of adolescent gender and informant on self-control could not be tested because these categories did not contain enough studies. Results are presented in Table 9. Regarding the moderators of theoretical interest, none of them was significant, including type of parenting (positive, negative, relationship), cultural characteristics (ethnicity, Hofstede's individualism), and parent gender (maternal parenting, paternal parenting, both parents).

In relation to the moderators of methodological interest, when including the report informant of parenting measure and consistency of informants, only the report informant of parenting measure yielded significant results, $Q_{F}(286)=1,999.973, p<.001, F(3,286)=$ $7.075, p<.001$.
Significant moderators. Follow-up analyses were conducted to further analyze the significant moderators. The results are summarized in Table 10 . With respect to the report informant of parenting measure, we found that effect sizes of the relation between self-control and parenting were all significant when parenting was assessed using adolescent self-report, other-report, observation, and composite measures. A follow-up comparison indicated that effect sizes of studies using composite measures to assess parenting were significantly larger than those using self-report, other-report, or observation. Effect sizes of studies using self-report were significantly larger than those using other-report. No multiple moderator analyses were applied because only one moderator was significant.

Publication bias. A funnel plot (illustrated in Fig. 6) and regression test indicated publication bias $(z=3.694$, $p<.001)$. Accordingly, the trim-and-fill procedure was applied to take publication bias into account, resulting in an adjusted effect size of $E S_{z}=0.153, S E=0.007,95 \%$ $\mathrm{CI}=[0.139,0.167], p<.001, r=.152,95 \% \mathrm{CI}=[.138, .165]$.

Table 9. Longitudinal Association for Self-Control Measured First and Parenting Measured Later: The $Q_{E}$ Statistics Testing Residual Heterogeneity and the Omnibus to Test the Effect of the Moderators

\begin{tabular}{lcccc}
\hline Moderator & $Q_{E}(d f)$ & $p$ & Omnibus test & $p$ \\
\hline Moderators of theoretical interest & & & & \\
$\quad$ Ethnicity of adolescents & $1,881.894(246)$ & $<.001$ & $F(5,246)=1.303$ & .263 \\
$\quad$ Hofstede's individualism & $2,253.226(289)$ & $<.001$ & $F(1,289)=0.323$ & .570 \\
$\quad$ Type of parenting & $2,263.721(288)$ & $<.001$ & $F(2,288)=0.122$ & .885 \\
$\quad$ Gender of parents & $2,264.139(287)$ & $<.001$ & $F(2,287)=2.354$ & .097 \\
Methodological characteristics & & & & \\
$\quad$ Report informant of parenting measure & $1,999.973(286)$ & $<.001$ & $F(3,286)=7.075$ & $<.001$ \\
$\quad$ Consistency of informants & $2,231.286(289)$ & $<.001$ & $F(1,289)=1.100$ & .295 \\
\hline
\end{tabular}




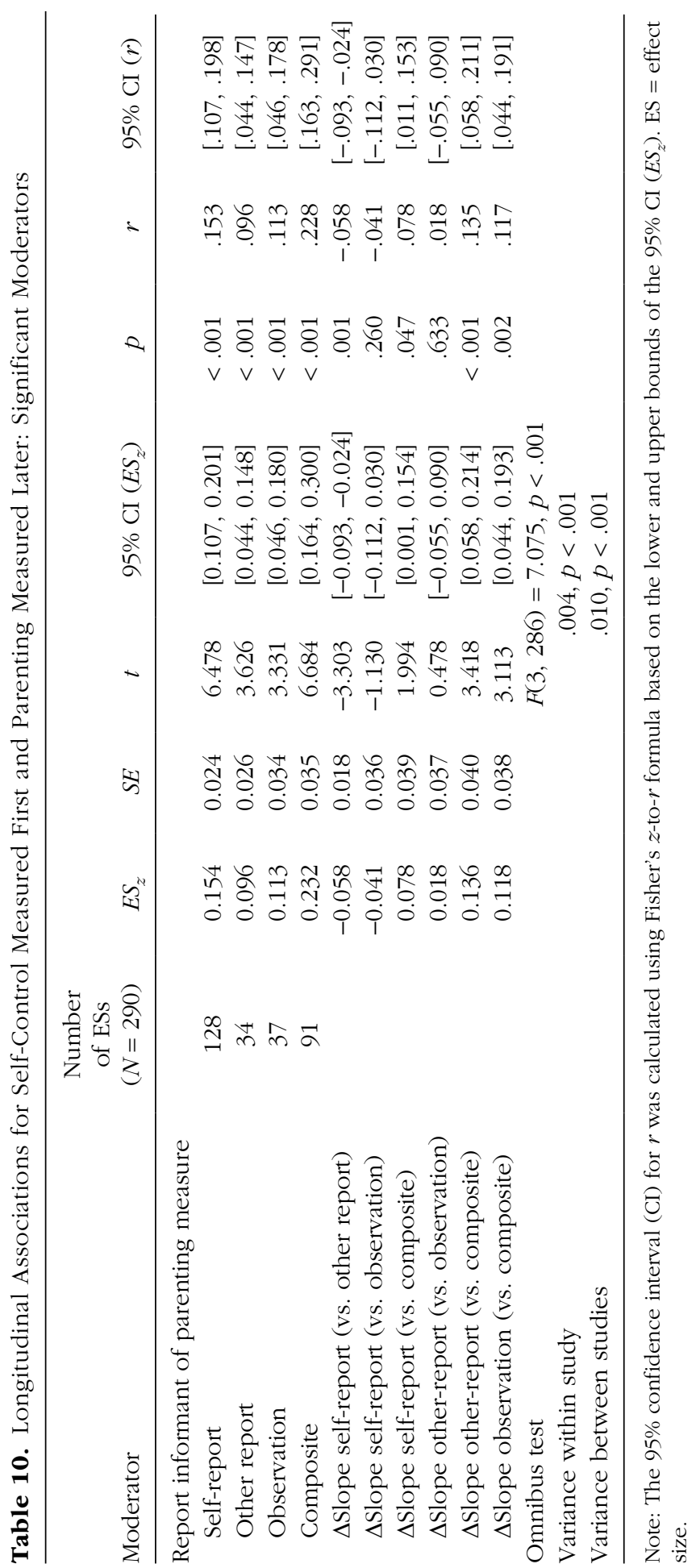


Table 11. Summary of Overall Effects and Results of Moderation Analyses Based on Multiple Moderator Tests

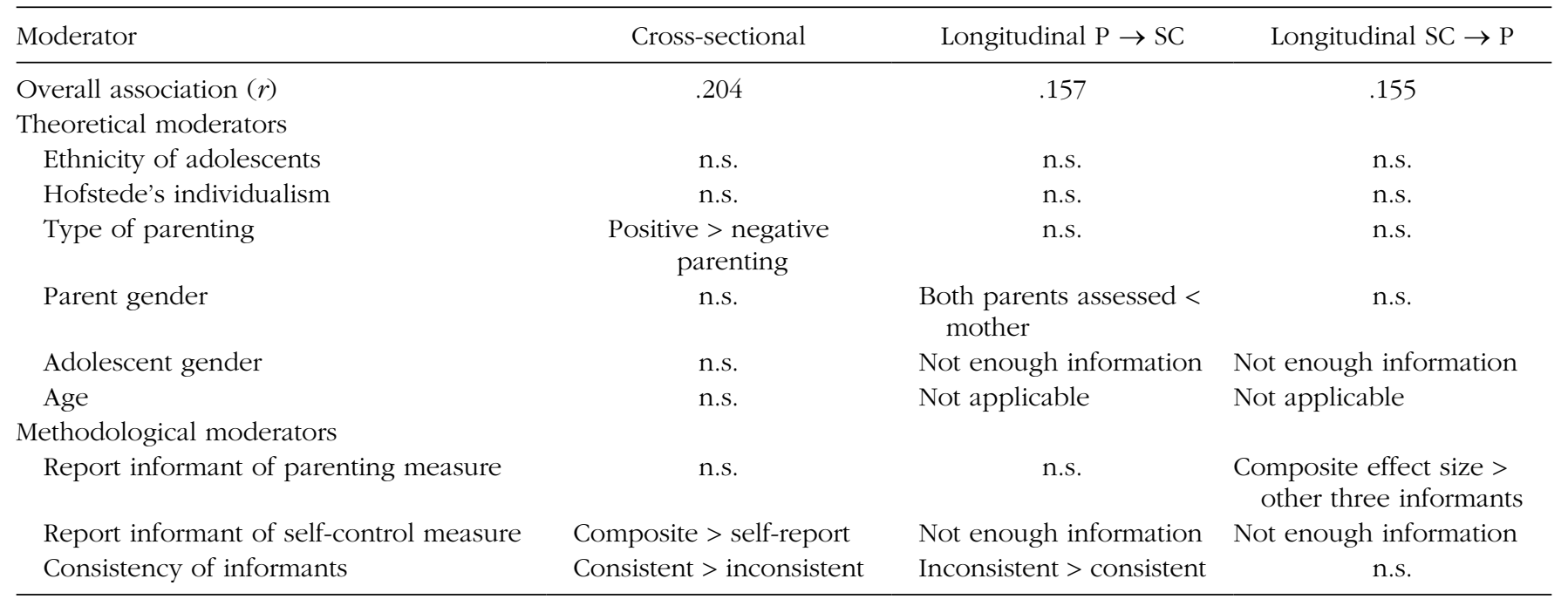

Note: $\mathrm{P}=$ parenting; $\mathrm{SC}=$ self-control. For longitudinal studies in which self-control was measured first and parenting was measured some time later $(\mathrm{SC} \rightarrow \mathrm{P}$ ), the summary is based on the results of individual moderation tests; no multiple-moderator test was run because there was a single significant moderator.

\section{Summary of results}

To let readers have a straightforward view of the results, we have summarized the overall cross-sectional and longitudinal associations and the significance of theoretical and methodological moderators in Table 11.

\section{Auxiliary analyses}

Effect size for the association between specific parenting dimensions and self-control. Although we subcategorized our parenting dimensions into the categories positive parenting, negative parenting, and parentchild relationships, we realize that the investigation of subcategories of parenting presented in Table 1 could be of interest to the field. We therefore provide free online access to our data, with details on specific parenting dimensions and analytic scripts. These data include detailed explanations so that researchers can easily add their data and/or further analyze the association between specific parenting dimensions and self-control and potential moderators influencing this association. Exploratory analyses between specific parenting dimensions (e.g., authoritative, supervision, negative control, attachment) and self-control from cross-sectional and longitudinal associations are presented in Table S1 in the Supplemental Material. As shown in the table, effect sizes of most subcategories of parenting were significantly related to selfcontrol with few exceptions (e.g., longitudinal association from positive expressivity or conflict to self-control). In addition, the magnitudes of these effect sizes vary to some extent. For instance, "relationship" is the parenting subcategory related to self-control with the largest effect sizes in both cross-sectional and longitudinal associations, whereas "harshness" and "conflict" had the smallest effect sizes for cross-sectional and longitudinal associations, respectively. For the longitudinal effect of self-control on parenting, relationship and harshness are most and least likely to be affected by adolescents' previous levels of self-control, respectively.

Power analysis. Table 12 displays the sample size needed to detect the average correlation found by this study at different levels of statistical power. For instance, the power analysis indicated that, to detect an effect size (r) of .204 at an $\alpha$ level of .05, a sample size of 186 participants was required for the study to have .80 power, 248 participants were required for .90 power, 306 participants were needed for .95 power, and 432 participants were needed for .99 power.

\section{Discussion}

Parenting has long been considered to be important to the development of self-control (Eisenberg et al., 2003; Eisenberg et al., 2005; Gottfredson \& Hirschi, 1990; Kochanska et al., 1995; Kopp, 1982; Sameroff, 2010). The current three-level meta-analysis is the first to synthesize the relation between broad types of parenting (i.e., positive parenting, negative parenting, and parentchild relationships) and self-control of adolescents aged 10 to 22 years. Analyses were based on 191 studies from 4 continents and included 1,540 effect sizes from a sample size of $N=164,459$. Our results showed that (a) parenting is concurrently and longitudinally associated with self-control throughout adolescence; (b) adolescent self-control significantly predicts subsequent parenting, and the predictive effect of parenting on 
Table 12. Recommendation of Sample Size

\begin{tabular}{|c|c|c|c|c|}
\hline \multirow[b]{2}{*}{ Design } & \multicolumn{4}{|c|}{ Power } \\
\hline & .80 & .90 & .95 & .99 \\
\hline 1. Cross-sectional design $(r=.204)$ & 186 & 248 & 306 & 432 \\
\hline 2. Longitudinal design: parenting measured first and self-control measured later $(r=.157)$ & 316 & 422 & 521 & 736 \\
\hline 3. Longitudinal design: self-control measured first and parenting measured later $(r=.155)$ & 324 & 433 & 535 & 755 \\
\hline
\end{tabular}

Note: In all cases, $\alpha$ was set at .05 with a two-tailed test. G*Power (Version 3.1.9.2; test family: exact; statistical test: correlation: bivariate normal model).

self-control and the one of self-control on parenting show similar magnitude; and (c) the relations between parenting and self-control (for both directions) are largely equal across cultures, ethnicities, parent and adolescent gender, and age, whereas the associations are moderated by a few methodological factors, such as report informant consistency. These findings provide a rich description of whether and how self-control and parenting are related across the entire period of adolescence.

Despite the changes that occur in adolescence such as eminent independence from parents and more investment in peer and romantic relationships (Connolly, Craig, Goldberg, \& Pepler, 2004; Nickerson \& Nagle, 2005; Song et al., 2009), our results showed that parenting was related to self-control in adolescence both concurrently and longitudinally. Speaking to the robustness of our findings, the effect sizes for the parent-child relationship-self-control association were similar to those reported in a recent meta-analysis focusing on the attachment security status and its relation with effortful control in children and adolescents up to the age of 18 years (Pallini et al., 2018).

Beyond the influence of parenting on self-control, our findings based on longitudinal studies also revealed a significant effect of adolescent self-control on subsequent parenting. Previous research has assumed an evocative effect from child's outcomes to parenting (e.g., Kochanska et al., 1995; Silverman \& Ragusa, 1990; Sameroff, 2010), but existing findings were inconsistent (e.g., J. Lee, Yu, \& Choi, 2012; Meldrum, Young, Hay, \& Flexon, 2012; Moilanen et al., 2015). The current findings, based on 291 effect sizes, provide support for the notion that adolescent self-control at a given time point does affect subsequent parenting behaviors, such that high self-control leads to more positive parenting, less negative parenting, and a better positive parentadolescent relationship. Low self-control, in contrast, is linked to diminished use of positive parenting (e.g., warmth, support) and increased use of negative parenting (e.g., physical and coercive punishment) and gives rise to a more negative parent-child relationship. We did not find a significant difference in the magnitude of the effect of parenting on adolescent self-control and the effect of adolescent self-control on parenting, which is consistent with developmental theories underpinning the importance of bidirectional interactions between adolescents and their (parental) environment for adolescent development (Bandura, 1999; Bronfenbrenner, 1979; Sameroff, 2010; Plomin et al., 1977). The current results revealed that, throughout adolescence, parenting continues to affect the development of adolescent selfcontrol and adolescent self-control continues to affect parenting.

The associations tested were found to be moderated by a few-mainly methodological-factors (see summary in Table 11). However, moderators for crosssectional studies did not necessarily extend to longitudinal studies (e.g., type of parenting) and vice versa (e.g., parent gender). Moreover, for some moderators, there was not enough information to detect their effects (e.g., adolescent gender for longitudinal studies). Some moderators (e.g., consistency of report informants) even showed contradictory moderating effects for cross-sectional and longitudinal studies. For example, in cross-sectional studies effect sizes were larger for studies using consistent rather than inconsistent report informants, but in longitudinal studies $(\mathrm{SC} \rightarrow \mathrm{P})$ effect sizes were larger for studies using inconsistent rather than consistent report informants.

On the basis of cross-sectional studies, which made up more than half of the total effect sizes, the relation between parenting and self-control tended to be stronger when parenting was positive than when parenting was negative, when self-control was measured using multiple report informants compared with a single report informant, and when informants of parenting and self-control were consistent.

Given the small moderating effects, our results suggest that the inconsistent findings regarding the association between parenting and self-control in the past may be largely due to methodological artifacts rather than theoretical misspecifications. In addition, we found a 
publication bias in longitudinal but not in cross-sectional studies, which may be a reason why moderators between cross-sectional and longitudinal studies were not entirely consistent, given that publication bias may also influence the estimates of between-study variance (Jackson, 2006).

\section{Theoretical implications}

The current results bear several theoretical implications. First, a number of theories and theoretical perspectives (e.g., attachment theory, the general theory of crime, the development of self-control and conscience, the unified theory of development) propose that good parenting is a crucial source of self-control in children (Bowlby, 1969; Gottfredson \& Hirschi, 1990; Kochanska, 1993; Kopp, 1982; Sameroff, 2010). The current findings imply that this proposition extends to adolescence and show that positive parenting and good parent-child relationships continue to play an essential role in shaping the individual differences in self-control from early to late adolescence; conversely, negative parenting and poor parent-child relationships continue to hamper adolescents' self-control. Second, the importance of parenting on adolescent self-control is largely equivalent across different cultures, ethnicities, and adolescent and parent gender. This suggests that the abovementioned theories and viewpoints regarding the influence of parenting on self-control are generally applicable across different demographic backgrounds, thus demonstrating their cross-cultural validity. Third, these theories and perspectives mainly focus on the parental effect on adolescents' self-control and disregard the examination of the evocative effect, as pointed out by some scholars (Lerner, 2002; Tiberio et al., 2016; Vazsonyi \& Huang, 2010). The current results demonstrated both parent and child effects regarding the relations between parenting and self-control, which suggests that the existing theories may need to take both parent and child effects into consideration to better capture the dynamic relation between parenting and self-control in adolescents.

\section{Limitations}

The current findings should be interpreted with caution. First, the sample size for the cross-sectional analyses was much larger than the sample size for the longitudinal analyses, resulting in more powerful analyses for the former. Because of this power issue, not all moderators could be tested in the longitudinal analyses.

Second, our meta-analysis included only communitybased adolescent samples, and its results may not be generalized to clinical samples (e.g., diagnosed with attentional disorder and hyperactivity disorder, autism spectrum disorder, diabetes) or samples with specific characteristics proposed to be related to self-control (e.g., prisoners, drug addicts).

Third, we acknowledge that the results based on the longitudinal studies refer to lagged associations or effects but not to changes in self-control or parenting because we did not control for the baseline levels of these constructs for two reasons. Conceptually, most longitudinal studies control not only for baseline levels of self-control or parenting but also for other covariates (e.g., child gender, age). This makes the $\beta$ coefficients noncomparable across studies. Methodologically, although we are aware that some new techniques, such as metaSEM (M. W. L. Cheung, 2015), have the potential to control for the target construct in a meta-analysis, the current version of such techniques is not as able to deal with dependency problems as a multilevel metaanalysis (the one applied in the current study). Not dealing with dependency problems possibly sacrifices much information, reduces statistical power, and even leads to biased results (Hox et al., 2010; Van den Noortgate et al., 2013). Nevertheless, we encourage scholars in the future to revisit this issue when more sophisticated statistical approaches evolve.

Last, the "similar effect size" for the longitudinal effect of parenting on adolescent self-control and the one of adolescent self-control on parenting refers to the effect sizes before adjusting for publication bias. After taking publication bias into account, the magnitudes of the two effect sizes appeared different. However, the trim-and-fill approach is controversial because it imputes nonexisting effect sizes (Sutton et al., 2000), and effect sizes as a result of such analyses cannot be convincingly compared. Therefore, this result should be interpreted with caution and take publication bias and the limitation of the trim-and-fill approach into consideration.

\section{Future Direction}

The current study bears important implications for future research. First, the included studies came from several continents, and the sample size was large (> 160,000). However, a majority of the effect sizes (62\%) were retrieved from studies conducted in the United States, and no or few eligible effect sizes were based on studies from African, South American, Southeastern Asian, Central Asian, and Eastern European regions. To further strengthen the current findings, we encourage scholars to integrate findings published in a wider variety of languages into our open-access data set. Doing so will eventually accumulate more effect sizes from a more varied population, which allows scholars to test 
a wider range of moderators and to achieve results with greater generalizability and higher robustness. Considering the fact that our data and scripts are freely accessible online, extending our results with international data is feasible. This also provides opportunities for other scholars who have different theoretical preferences to categorize parenting such as warmth, behavioral control, and autonomy support (e.g., Prinzie, Stams, Deković, Reijntjes, \& Belsky, 2009) to analyze our data for different research questions and facilitates an update of the meta-analysis in the future. Second, among the studies included in this meta-analysis, many assessed parenting in general without separately referring to mothers or fathers. Although mothers' parenting and fathers' parenting often show medium-to-high correlations (e.g., J. B. Li et al., 2015; Ng-Knight, Shelton, Frederickson, McManus, \& Rice, 2018; Nie et al., 2016; Özdemir et al., 2013), examining parenting for mothers and fathers separately would be promising. It may allow researchers to identify the similarities and differences between maternal and paternal influences on adolescent self-control. Future research regarding this issue may want to separately explore the effect of mothers and fathers.

Third, the magnitude of the effect sizes suggests that adolescent self-control is influenced by multiple socialization agents. For example, peers and teachers are also potential socializing agents steering adolescents' selfcontrol (e.g., Alvarez-Rivera \& Fox, 2010; Meldrum, 2008; Turner, Piquero, \& Pratt, 2005). A recent metaanalysis shows that the heritability of self-control is $60 \%$, highlighting that individual differences in selfcontrol are the result not only of socializing factors but also of biological factors (Willems, Boesen, Li, Finkenauer, \& Bartels, 2019). However, much of the literature to date evaluates the development of selfcontrol as a result of environmental socialization. Incorporating biological studies is necessary to paint a more complete picture of individual differences in self-control. Future studies applying genetically sensitive designs are particularly promising, as these allow researchers to investigate whether the association between parenting and self-control is genetically based, environmentally based, or a combination of these (Willems, de Zeeuw, et al., 2019).

Last, this meta-analysis provided overall effect sizes for cross-sectional and longitudinal associations between parenting and self-control. This knowledge on the average effect size allows us to provide additional recommendations for future research. Specifically, it provides information on the number of participants necessary to detect the current findings. Doing so will allow researchers to gauge the appropriate level of conservatism or liberalism they prefer when recruiting participants and helps researchers make the most of their time and resources. Table 12 summarizes sample sizes to achieve the correlation coefficients transformed back from effect sizes at four levels of power with an alpha level of .05. It should be noted that these sample sizes are estimated for bivariate correlations. If researchers wish to conduct other statistical analyses in future studies, they may need to recalculate the sample size on the basis of the effect sizes found in this study. However, this can be easily implemented in G*Power or using other approaches (e.g., Monte Carlo simulation).

\section{Concluding Remarks}

Ill decisions and reckless behaviors due to low selfcontrol in adolescence are at the cost of individual physical and psychosocial functioning as well as social security, both concurrently and longitudinally (Caspi et al., 2016; Moffitt et al., 2011). The current study suggests that parenting significantly contributes to selfcontrol in adolescents aged 10 to 22 years. It also suggests that adolescent self-control shows a significant lagged effect on subsequent parenting. These relationships are largely equal across cultures, ethnicities, parent and child gender, and age of adolescents, and only a few (mainly methodological) factors moderate this relationship. Our findings provide further evidence for the importance of considering the continuous and dynamic interplay of the development of self-control and environment (parenting or parent-child relationship) across the adolescent period.

\section{Action Editor}

Laura A. King served as action editor for this article.

\section{Author Contributions}

J.-B. Li and Y. E. Willems contributed equally to this manuscript and are co-first authors. All of the authors approved the final manuscript for submission.

\section{ORCID iD}

Jian-Bin Li (iD https://orcid.org/0000-0002-8995-3304

\section{Declaration of Conflicting Interests}

The author(s) declared that there were no conflicts of interest with respect to the authorship or the publication of this article.

\section{Funding}

J.-B. Li is supported by the Faculty of Education and Human Development's Internationalization and Exchange Research Scheme of the Education University of Hong Kong. Y. Willems 
is supported by Dutch Research Council (NWO) Research Talent Grant 406-15-132 and by the Amsterdam Public Health personalized medicine travel grant. M. Bartels is financially supported by a Vrije Universiteit Amsterdam research chair position; the Aggression in Children: Unraveling GeneEnvironment Interplay to Inform Treatment and Intervention Strategies project, which receives funding from the European Union Seventh Framework Program (FP7/2007-2013) under grant agreement 602768; and the Marie Skłodowska-Curie ACTIONS Innovative Training Network CAPICE (Childhood and Adolescence Psychopathology: Unravelling the Complex Etiology by a Large Interdisciplinary Collaboration in Europe; H2020-MSCA-ITN-2016 721567-CAPICE).

\section{Supplemental Material}

Additional supporting information can be found at http:// journals.sagepub.com/doi/suppl/10.1177/1745691619863046

\section{Notes}

1. We preregistered this research at https://aspredicted.org/ gs2zj.pdf.

2. Adolescence is a period connecting early childhood and emerging adulthood. Although the adolescent period is considered to begin around the age of 10 years, characterized by the onset of puberty (United Nations Children's Fund, 2011), the end of this developmental period receives less consensus. Recent neuroscientific research has suggested that 21 to 22 years of age could be the cutoff when adolescents become adults (A. O. Cohen et al., 2016). Thus, this study focuses on early adolescence to late adolescence ranging from 10 to 22 years.

3. Attachment during adolescence is usually understood or measured as a close parent-child relationship and can be considered as the continuum of childhood attachment rather than the attachment dimensions as by Bowlby \& Ainsworth (Armsden \& Greenberg, 1987). In this study we therefore used the term "parent-child relationship" instead of "parent-child attachment," but in the literature search and coding we categorized relationship and attachment as the same subtype, namely "parent-child relationship."

4. In the preregistration, we did not include the search term effortful control because we expected our initial terms to yield articles on effortful control. However, during an initial trial of our search, we noticed that important effortful-control articles were missing. As a result, we repeated the search including effortful and control as separate search terms to make our search more inclusive. In addition, M. Deković and M. Bartels were added as co-authors of the article considering their invaluable insights and collaborations later in the project. Given that we included existing data sets, we did not seek ethical approval in the current work.

5. Key words such as child and youth were included in the search to ensure the inclusion of longitudinal studies focusing on early or middle childhood but possibly also including longitudinal correlations up to adolescence.

6. The Fisher's transformation of $r$ was done using the following formula: $E S_{Z r}=\frac{1}{2} \log _{e}\left[\frac{1+r}{1-r}\right]$. Any $E S_{Z r}$ can be transformed back into standard correlation form using the inverse of the $E S_{Z r}$ transformation using the following formula: $r=\frac{e^{2 E S_{Z r}}-1}{e^{2 E S_{Z r}}+1}$ (see
Field, 2001; Lipsey \& Wilson, 2001).

7. The overall sample size was calculated by summing the largest sample size within unique samples when more than one effect size was collected.

8. We were also interested to see whether age could show a nonlinear pattern. To this end, we checked whether the squared and/or cubical age served as a significant moderator. However, our results showed no significant quadratic, $Q_{\mathrm{E}}(830)=$ $10,384.754, p<.001, F(1,830)=1.698, p=.193$, or the cubic pattern, $Q_{\mathrm{E}}(830)=10,361.892, p<.001, F(1,830)=1.693, p=.194$.

\section{References}

References marked with an asterisk indicate studies included in the meta-analysis.

*Aaltonen, M., \& Salmi, V. (2013). Versatile delinquents or specialized pirates? A comparison of correlates of illegal downloading and traditional juvenile crime. Journal of Scandinavian Studies in Criminology and Crime Prevention, 14, 188-195. doi:10.1080/14043858.2013.837267

*Abedini, Y., Zamani, B. E., Kheradmand, A., \& Rajabizadeh, G. (2012). Impacts of mothers' occupation status and parenting styles on levels of self-control, addiction to computer games, and educational progress of adolescents. Addiction \& Health, 4, 102-110. doi:10.1016/S09249338(15)30399-0

*Agbaria, Q., Hamama, L., Orkibi, H., Gabriel-Fried, B., \& Ronen, T. (2016). Multiple mediators for peer-directed aggression and happiness in Arab adolescents exposed to parent-child aggression. Child Indicators Research, 9, 785-803. doi:10.1007/s12187-015-9346-y

Allan, N. P., Hume, L. E., Allan, D. M., Farrington, A. L., \& Lonigan, C. J. (2014). Relations between inhibitory control and the development of academic skills in preschool and kindergarten: A meta-analysis. Developmental Psychology, 50, 2368-2379. doi:10.1037/a0037493

Allan, N. P., \& Lonigan, C. J. (2011). Examining the dimensionality of effortful control in preschool children and its relation to academic and socioemotional indicators. Developmental Psychology, 47, 905-915. doi:10.1037/ a0023748

*Alvarez-Rivera, L. L., \& Fox, K. A. (2010). Institutional attachments and self-control: Understanding deviance among Hispanic adolescents. Journal of Criminal Justice, 38, 666-674. doi:10.1016/j.jcrimjus.2010.04.040

*Anderson, S. A., Sabatelli, R. M., \& Kosutic, I. (2007). Families, urban neighborhood youth centers, and peers as contexts for development. Family Relations, 56, 346-357. doi:10.1111/j.1741-3729.2007.00464.x

*Ardestani, M., Niknami, S., Hidarnia, A., \& Hajizadeh, E. (2015). Predictors of physical activity among adolescent girl students based on the social cognitive theory. Journal of Research in Health Sciences, 15, 223-227.

Armsden, G. C., \& Greenberg, M. T. (1987). The inventory of parent and peer attachment: Individual differences and their relationship to psychological wellbeing 
in adolescence. Journal of Youth and Adolescence, 16, 427-454. doi:10.1007/BF02202939

Arnett, J. (1992). Reckless behavior in adolescence: A developmental perspective. Developmental Review, 12, 339373. doi:10.1016/0273-2297(92)90013-R

Arnett, J. (1999). Adolescent storm and stress, reconsidered. American Psychologist, 54, 317-326. doi:10.1037/0003066X.54.5.317

Arroyo, J., Payne, K. K., Brown, S. L., \& Manning, W. D. (2013). Crossover in median age at first marriage and first birth: Thirty years of change. Bowling Green, $\mathrm{OH}$ : National Center for Family and Marriage Research.

Assink, M., \& Wibbelink, C. J. (2016). Fitting three-level meta-analytic models in R: A step-by-step tutorial. The Quantitative Methods for Psychology, 12, 154-174. doi:10.20982/tqmp.12.3.p154

*Baardstu, S., Karevold, E. B., \& von Soest, T. (2017). Childhood antecedents of Agreeableness: A longitudinal study from preschool to late adolescence. Journal of Research in Personality, 67, 202-214. doi:10.1016/j .jrp.2016.10.007

*Baker, C. N., \& Hoerger, M. (2012). Parental child-rearing strategies influence self-regulation, socio-emotional adjustment, and psychopathology in early adulthood: Evidence from a retrospective cohort study. Personality and Individual Differences, 52, 800-805. doi:10.1016/j .paid.2011.12.034

*Baker, J. O. (2010). The expression of low self-control as problematic drinking in adolescents: An integrated control perspective. Journal of Criminal Justice, 38, 237-244. doi:10.1016/j.jcrimjus.2010.02.011

*Bakker, M. P., Ormel, J., Verhulst, F. C., \& Oldehinkel, A. J. (2011). Adolescent family adversity and mental health problems: The role of adaptive self-regulation capacities. The TRAILS study. Journal of Abnormal Child Psychology, 39, 341-350. doi:10.1007/s10802-010-9470-6

Bandura, A. (1999). A social cognitive theory of personality. In L. Pervin \& O. John (Eds.), Handbook of personality (2nd ed., pp. 154-196). New York, NY: Guilford Press.

*Barnes, J. C., \& Morris, R. G. (2012). Young mothers, delinquent children: Assessing mediating factors among American youth. Youth Violence and Juvenile Justice, 10, 172-189. doi:10.1177/1541204011423260

Baumeister, R. F., Vohs, K. D., \& Tice, D. M. (2007). The strength model of self-control. Current Directions in Psychological Science, 16, 351-355. doi:10.1111/j.14678721.2007.00534.x

Baumrind, D. (1991). The influence of parenting styles on adolescent competence and substance use. Journal of Early Adolescence, 11, 56-95. doi:10.1177/0272431691111004

*Bayraktar, F., Machackova, H., Dedkova, L., Cerna, A., \& Sevcikova, A. (2015). Cyberbullying: The discriminant factors among cyberbullies, cybervictims, and cyberbully-victims in a Czech adolescent sample. Journal of Interpersonal Violence, 30, 3192-3216. doi:10.1177/0886260514555006

*Beaver, K. M., Ferguson, C. J., \& Lynn-Whaley, J. (2010). The association between parenting and levels of self-control: A genetically informative analysis. Criminal Justice and Behavior, 37, 1045-1065. doi:10.1177/0093854810374919
Beaver, K. M., Wright, J. P., \& DeLisi, M. (2007). Self-control as an executive function: Reformulating Gottfredson and Hirschi's parental socialization thesis. Criminal Justice and Behavior, 34, 1345-1361. doi:10.1177/0093854807302049

Begg, C. B. (1994). Publication bias. In H. Cooper \& L. V. Hedges (Eds.), The handbook of research synthesis (pp. 399-409). New York, NY: Russell Sage Foundation.

Belsky, J. (1984). The determinants of parenting: A process model. Child Development, 55, 83-96. doi:10.2307/1129836

*Berzonsky, M. D., Branje, S. J., \& Meeus, W. (2007). Identity-processing style, psychosocial resources, and adolescents' perceptions of parent-adolescent relations. The Journal of Early Adolescence, 27, 324-345. doi:10.1177/0272431607302006

Bowlby, J. (1969). Attachment and loss. Vol. 1: Attachment. New York, NY: Basic Books.

Boyer, T. (2006). The development of risk-taking: A multiperspective review. Developmental Review, 26, 291-345. doi:10.1016/j.dr.2006.05.002

*Bradley, R. H., \& Corwyn, R. (2013). From parent to child to parent . . . Paths in and out of problem behavior. Journal of Abnormal Child Psychology, 41, 515-529. doi:10.1007/ s10802-012-9692-x

*Bradley, R. H., \& Corwyn, R. F. (2007). Externalizing problems in fifth grade: Relations with productive activity, maternal sensitivity, and harsh parenting from infancy through middle childhood. Developmental Psychology, 43, 1390-1401. doi:10.1037/0012-1649,43.6.1390

*Brauer, J. R. (2017). Cultivating conformists or raising rebels? Connecting parental control and autonomy support to adolescent delinquency. Journal of Research on Adolescence, 27, 452-470. doi:10.1111/jora.12283

Bridgett, D. J., Burt, N. M., Edwards, E. S., \& Deater-Deckard, K. (2015). Intergenerational transmission of self-regulation: A multidisciplinary review and integrative conceptual framework. Psychological Bulletin, 141, 602-654. doi: $10.1037 / \mathrm{a} 0038662$

*Brody, G. H., Dorsey, S., Forehand, R., \& Armistead, L. (2002). Unique and protective contributions of parenting and classroom processes to the adjustment of African American children living in single-parent families. Child Development, 73, 274-286. doi:10.1111/1467-8624.00405

*Brody, G. H., \& Ge, X. (2001). Linking parenting processes and self-regulation to psychological functioning and alcohol use during early adolescence. Journal of Family Psychology, 15, 82-94. doi:10.1037/0893-3200.15.1.82

*Brody, G. H., McBride Murry, V., McNair, L., Chen, Y. F., Gibbons, F. X., Gerrard, M., \& Ashby Wills, T. (2005). Linking changes in parenting to parent-child relationship quality and youth self-control: The Strong African American Families Program. Journal of Research on Adolescence, 15, 47-69. doi:10.1111/j.1532-7795.2005.00086.x

*Brody, G. H., Murry, V. M., Kim, S., \& Brown, A. C. (2002) Longitudinal pathways to competence and psychological adjustment among African American children living in rural single-parent households. Child Development, 73 , 1505-1516. doi:10.1111/1467-8624.00486

*Brody, G. H., Stoneman, Z., \& Flor, D. (1995). Linking family processes and academic competence among rural African 
American youths. Journal of Marriage and the Family, 57, 567-579. doi:10.2307/353913

*Brody, G. H., Stoneman, Z., Smith, T., \& Gibson, N. M. (1999). Sibling relationships in rural African American families. Journal of Marriage and the Family, 61, 10461057. doi:10.2307/354023

Bronfenbrenner, U. (1979). The ecology of human development. Cambridge, MA: Harvard University Press.

*Buck, K. A. (2014). Sensitivity, child regulatory processes, and naturally occurring declines in antisocial behavior across childhood. Journal of Family Psychology, 28, 925935. doi: $10.1037 /$ fam0000029

*Buck, K. A. (2015). Understanding adolescent psychopathic traits from early risk and protective factors: Relations among inhibitory control, maternal sensitivity, and attachment representation. Journal of Adolescence, 44, 97-105. doi:10.1016/j.adolescence.2015.07.009

Buist, K. L., Deković, M., Meeus, W., \& van Aken, M. A. (2002). Developmental patterns in adolescent attachment to mother, father and sibling. Journal of Youth and Adolescence, 31, 167-176. doi:10.1023/A:1015074701280

*Burt, C. H., Simons, R. L., \& Simons, L. G. (2006). A longitudinal test of the effects of parenting and the stability of self-control: Negative evidence for the general theory of crime. Criminology, 44, 353-396. doi:10.1111/j.17459125.2006.00052.x

Buyukcan-Tetik, A., Finkenauer, C., Siersema, M., Vander Heyden, K., \& Krabbendam, L. (2015). Social relations model analyses of perceived self-control and trust in families. Journal of Marriage and Family, 77, 209-223. doi:10.1111/jomf.12154

*Caprara, G. V., Gerbino, M., Paciello, M., Di Giunta, L., \& Pastorelli, C. (2010). Counteracting depression and delinquency in late adolescence. European Psychologist, 15, 34-48. doi:10.1027/1016-9040/a000004

Casey, B. J., Jones, R. M., \& Hare, T. A. (2008). The adolescent brain. Annals of the New York Academy of Sciences, 1124 , 111-126. doi:10.1196/annals.1440.010

Caspi, A., Houts, R. M., Belsky, D. W., Harrington, H., Hogan, S., Ramrakha, S., \& Moffitt, T. E. (2016). Childhood forecasting of a small segment of the population with large economic burden. Nature Human Behaviour, 1, Article 0005. doi:10.1038/s41562-016-0005

Caspi, A., \& Roberts, B. W. (2001). Personality development across the life course: The argument for change and continuity. Psychological Inquiry, 12, 49-66. doi:10.1207/ S15327965PLI1202_01

Cassidy, J. (1994). Emotion regulation: Influences of attachment relationships. Monographs of the Society for Research in Child Development, 59, 228-249. doi:10.1111/j.1540-5834.1994 .tb01287.x

Chao, R. K. (1994). Beyond parental control and authoritarian parenting style: Understanding Chinese parenting through the cultural notion of training. Child Development, 65 , 1111-1119. doi:10.1111/j.1467-8624.1994.tb00806.x

Chapple, C. L., Vaske, J., \& Hope, T. L. (2010). Sex differences in the causes of self-control: An examination of mediation, moderation, and gendered etiologies. Journal of Criminal Justice, 38, 1122-1131. doi:10.1016/j.jcrim jus.2010.08.004

*Chen, J. K., \& Avi Astor, R. (2010). School violence in Taiwan: Examining how Western risk factors predict school violence in an Asian culture. Journal of Interpersonal Violence, 25, 1388-1410. doi:10.1177/0886260509354576

Cheung, M. W. L. (2014). Modeling dependent effect sizes with three-level meta-analyses: A structural equation modeling approach. Psychological Methods, 19, 211-229. doi: $10.1037 / \mathrm{a} 0032968$

Cheung, M. W. L. (2015). Meta-analysis: A structural equation modeling approach. Hoboken, NJ: Wiley.

*Cheung, N. W. T., \& Cheung, Y. W. (2008). Self-control, social factors, and delinquency: A test of the general theory of crime among adolescents in Hong Kong. Journal of Youth and Adolescence, 37, 412-430. doi:10.1007/s10964007-9218-y

*Cheung, N. W. T., \& Cheung, Y. W. (2010). Strain, self-control, and gender differences in delinquency among Chinese adolescents: Extending general strain theory. Sociological Perspectives, 53, 321-345. doi:10.1525/sop.2010.53.3.321

*Cho, J., Kogan, S. M., \& Brody, G. H. (2016). Genetic moderation of transactional relations between parenting practices and child self-regulation. Journal of Family Psychology, 30, 780-790. doi:10.1037/fam0000228

*Chun, J., \& Chung, I.-J. (2013). Gender differences in factors influencing smoking, drinking, and their co-occurrence among adolescents in South Korea. Nicotine \& Tobacco Research, 15, 542-551. doi:10.1093/ntr/nts181

*Clark, D. A., Donnellan, M. B., Robins, R. W., \& Conger, R. D. (2015). Early adolescent temperament, parental monitoring, and substance use in Mexican-origin adolescents. Journal of Adolescence, 41, 121-130. doi:10.1016/j.ado lescence.2015.02.010

Cohen, A. O., Breiner, K., Steinberg, L., Bonnie, R. J., Scott, E. S., Taylor-Thompson, K., . . . Silverman, M. R. (2016). When is an adolescent an adult? Assessing cognitive control in emotional and nonemotional contexts. Psychological Science, 24, 549-562. doi:10.1177/0956797615627625

Collins, W. A., \& Steinberg, L. (2006). Adolescent development in interpersonal context: Socioemotional processes. In W. Damon \& N. Eisenberg (Eds.), Handbook of child psychology: Socioemotional processes (Vol. 4, pp. 10031067). Hoboken, NJ: Wiley.

Connolly, J., Craig, W., Goldberg, A., \& Pepler, D. (2004). Mixed-gender groups, dating, and romantic relationships in early adolescence. Journal of Research on Adolescence, 14, 185-207. doi:10.1111/j.1532-7795.2004.01402003.x

*Craig, J. M. (2016). Which bond matters more? Assessing the differential strengths of parental bonding measures on adolescent delinquency over time. Youth Violence and Juvenile Justice, 14, 225-242. doi:10.1177/1541204014565670

Crone, E. A., \& Dahl, R. E. (2012). Understanding adolescence as a period of social-affective engagement and goal flexibility. Nature Reviews Neuroscience, 13, 636-650. doi:10.1038/nrn3313

*Crossley, I. A., \& Buckner, J. C. (2012). Maternal-related predictors of self-regulation among low-income youth. 
Journal of Child and Family Studies, 21, 217-227. doi:10.1007/s10826-011-9465-0

*Crosswhite, J. M., \& Kerpelman, J. L. (2012). Parenting and children's self-control: Concurrent and longitudinal relations. Deviant Behavior, 33, 715-737. doi:10.1080/0163 9625.2011.647597

*Cui, L., Morris, A. S., Criss, M. M., Houltberg, B. J., \& Silk, J. S. (2014). Parental psychological control and adolescent adjustment: The role of adolescent emotion regulation. Parenting, 14, 47-67. doi:10.1080/15295192.2014.880018

Darling, N., \& Steinberg, L. (1993). Parenting style as context: An integrative model. Psychological Bulletin, 113, 487-496. doi:10.1037/0033-2909.113.3.487

*Davenport, E., Yap, M. B., Simmons, J. G., Sheeber, L. B., \& Allen, N. B. (2011). Maternal and adolescent temperament as predictors of maternal affective behavior during mother-adolescent interactions. Journal of Adolescence, 34, 829-839. doi:10.1016/j.adolescence.2011.02.003

Davis, M., Bilms, J., \& Suveg, C. (2017). In sync and in control: A meta-analysis of parent-child positive behavioural synchrony and youth self-regulation. Family Process, 56, 962-980. doi:10.1111/famp.12259

*del Castillo, J. A. G., Dias, P. C., Pérez, J. D., \& del Castillo López, Á. G. (2012). The mediating role of self-regulation in cigarette smoking and alcohol use among young people. Anales de Psicología/Annals of Psychology, 28, 1-10.

*Delker, B. C., Noll, L. K., Kim, H. K., \& Fisher, P. A. (2014). Maternal abuse history and self-regulation difficulties in preadolescence. Child Abuse \& Neglect, 38, 2033-2043. doi:10.1016/j.chiabu.2014.10.014

De Ridder, D. T., Lensvelt-Mulders, G., Finkenauer, C., Stok, F. M., \& Baumeister, R. F. (2012). Taking stock of self-control: A meta-analysis of how trait self-control relates to a wide range of behaviors. Personality and Social Psychology Review, 16, 76-99. doi:10.1177/1088868311418749

*Deschenes, E. P., \& Esbensen, F. A. (1999). Violence and gangs: Gender differences in perceptions and behavior. Journal of Quantitative Criminology, 15, 63-96. doi:10.1023/A:1007552105190

*Doan, S. N., Fuller-Rowell, T. E., \& Evans, G. W. (2012). Cumulative risk and adolescent's internalizing and externalizing problems: The mediating roles of maternal responsiveness and self-regulation. Developmental Psychology, 48, 1529-1539. doi:10.1037/a0027815

Doyle, A., \& Markiewicz, D. (2005). Parenting, marital conflict and adjustment from early- to mid-adolescence: Mediated by adolescent attachment style? Journal of Youth and Adolescence, 34, 97-110. doi:10.1007/s10964-005-3209-7

*Drake, K., Belsky, J., \& Fearon, R. M. P. (2014). From early attachment to engagement with learning in school: The role of self-regulation and persistence. Developmental Psychology, 50, 1350-1361. doi:10.1037/a0032779

Duckworth, A. L., \& Kern, M. L. (2011). A meta-analysis of the convergent validity of self-control measures. Journal of Research in Personality, 45, 259-268. doi:10.1016/j .jrp.2011.02.004

Duckworth, A. L., \& Seligman, M. E. (2005). Self-discipline outdoes IQ in predicting academic performance of adolescents. Psychological Science, 16, 939-944. doi:10.1111/j.1467-9280.2005.01641.x

Duckworth, A. L., \& Steinberg, L. (2015). Unpacking selfcontrol. Child Development Perspectives, 9, 32-37. doi:10 .1111/cdep. 12107

Duval, S., \& Tweedie, R. (2000a). A nonparametric "trim and fill" method of accounting for publication bias in metaanalysis. Journal of the American Statistical Association, 95, 89-98. doi:10.1080/01621459.2000.10473905

Duval, S., \& Tweedie, R. (2000b). Trim and fill: A simple funnel-plot-based method of testing and adjusting for publication bias in meta-analysis. Biometrics, 56, 455-463. doi:10.1111/j.0006-341X.2000.00455.x

Egger, M., Smith, G. D., Schneider, M., \& Minder, C. (1997). Bias in meta-analysis detected by a simple, graphical test. BMJ, 315(7109), 629-634. doi:10.1136/bmj.315.7109.629

*Eiden, R. D., Lessard, J., Colder, C. R., Livingston, J., Casey, M., \& Leonard, K. E. (2016). Developmental cascade model for adolescent substance use from infancy to late adolescence. Developmental Psychology, 52, 1619-1633. doi:10.1037/dev0000199

*Eisenberg, N., Hofer, C., Spinrad, T. L., Gershoff, E. T., Valiente, C., Losoya, S., ... Maxon, E. (2008). Understanding mother-adolescent conflict discussions: Concurrent and across-time prediction from youths' dispositions and parenting. Monographs of the Society for Research in Child Development, 73, 1-160. doi:10.1111/ j.1540-5834.2008.00470.x

*Eisenberg, N., Zhou, Q., Losoya, S. H., Fabes, R. A., Shepard, S. A., Murphy, B. C., .. . Cumberland, A. (2003). The relations of parenting, effortful control, and ego control to children's emotional expressivity. Child Development, 74, 875-895. doi:10.1111/1467-8624.00573

*Eisenberg, N., Zhou, Q., Spinrad, T. L., Valiente, C., Fabes, R. A., \& Liew, J. (2005). Relations among positive parenting, children's effortful control, and externalizing problems: A three-wave longitudinal study. Child Development, 76, 1055-1071. doi:10.1111/j.1467-8624.2005.00897.x

*Evans, S. Z., Simons, L. G., \& Simons, R. L. (2012). The effect of corporal punishment and verbal abuse on delinquency: Mediating mechanisms. Journal of Youth and Adolescence, 41, 1095-1110. doi:10.1007/s10964-012-9755-x

Fan, J., Flombaum, J. I., McCandliss, B. D., Thomas, K. M., \& Posner, M. I. (2003). Cognitive and brain consequences of conflict. NeuroImage, 18, 42-57. doi:10.1006/ nimg. 2002.1319

Farley, J. P., \& Kim-Spoon, J. (2014). The development of adolescent self-regulation: Reviewing the role of parent, peer, friend, and romantic relationships. Journal of Adolescence, 37, 433-440. doi:10.1016/j.adolescence.2014.03.009

Faul, F., Erdfelder, E., Lang, A. G., \& Buchner, A. (2007). G*Power 3: A flexible statistical power analysis program for the social, behavioral, and biomedical sciences. Behavior Research Methods, 39, 175-191. doi:10.3758/BF03193146

*Feldman, S. S., \& Brown, N. L. (1993). Family influences on adolescent male sexuality: The mediational role of self-restraint. Social Development, 2, 15-35. doi:10.1111/ j.1467-9507.1993.tb00002.x 
*Feldman, S. S., \& Wentzel, K. R. (1990). Relations among family interaction patterns, classroom self-restraint, and academic achievement in preadolescent boys. Journal of Educational Psychology, 82, 813-819. doi:10.1037/00220663.82.4.813

*Feldman, S. S., \& Wentzel, K. R. (1990). The relationship between parenting styles, sons' self-restraint, and peer relations in early adolescence. The Journal of Early Adolescence, 10, 439-454. doi:10.1177/0272431690104002

*Feldman, S. S., Wentzel, K. R., Weinberger, D. A., \& Munson, J. A. (1990). Marital satisfaction of parents of preadolescent boys and its relationship to family and child functioning. Journal of Family Psychology, 4, 213-234. doi:10.1037/0893-3200.4.2.213

*Ferdik, F. V., Wolfe, S. E., \& Blasco, N. (2014). Informal social controls, procedural justice and perceived police legitimacy: Do social bonds influence evaluations of police legitimacy? American Journal of Criminal Justice, 39, 471-492. doi:10.1007/s12103-013-9230-6

Ferguson, C. J., \& Brannick, M. T. (2012). Publication bias in psychological science: Prevalence, methods for identifying and controlling, and implications for the use of meta-analyses. Psychological Methods, 17, 120-128. doi: $10.1037 / \mathrm{a} 0024445$

Field, A. P. (2001). Meta-analysis of correlation coefficients: A Monte Carlo comparison of fixed- and randomeffects methods. Psychological Methods, 6, 161-180. doi:10.1037/1082-989X.6.2.161

Figner, B., Knoch, D., Johnson, E. J., Krosch, A. R., Lisanby, S. H., Fehr, E., \& Weber, E. U. (2010). Lateral prefrontal cortex and self-control in intertemporal choice. Nature Neuroscience, 13, 538-539. doi:10.1038/nn.2516

*Finkenauer, C., Engels, R., \& Baumeister, R. (2005). Parenting behaviour and adolescent behavioural and emotional problems: The role of self-control. International Journal of Behavioral Development, 29, 58-69. doi:10.1080/0165 0250444000333

Fisher, R. A. (1921). On the probable error of a coefficient of correlation deduced from a small sample. Metron, 1, 1-32.

*Fosco, G. M., Caruthers, A. S., \& Dishion, T. J. (2012). A six-year predictive test of adolescent family relationship quality and effortful control pathways to emerging adult social and emotional health. Journal of Family Psychology, 26, 565-575. doi:10.1037/a0028873

*Fosco, G. M., \& Grych, J. H. (2013). Capturing the family context of emotion regulation: A family systems model comparison approach. Journal of Family Issues, 34, 557578. doi:10.1177/0192513X12445889

*Fox, K. A., \& Bouffard, L. A. (2015). Violent victimization vulnerability: Testing a conceptual model of personality, social, and community factors. Deviant Behavior, 36, 910-934. doi:10.1080/01639625.2014.977201

*Frijns, T., Finkenauer, C., Vermulst, A. A., \& Engels, R. C. (2005). Keeping secrets from parents: Longitudinal associations of secrecy in adolescence. Journal of Youth and Adolescence, 34, 137-148. doi:10.1007/s10964-005-3212-z

Fu, A. S., \& Markus, H. R. (2014). My mother and me: Why tiger mothers motivate Asian Americans but not European
Americans. Personality and Social Psychology Bulletin, 40, 739-749. doi:10.1177/0146167214524992

*Furlong, M. J., You, S., Shishim, M., \& Dowdy, E. (2017). Development and validation of the Social Emotional Health Survey-Higher Education version. Applied Research in Quality of Life, 12, 343-367. doi:10.1007/ s11482-016-9464-9

Galla, B. M., \& Duckworth, A. L. (2015). More than resisting temptation: Beneficial habits mediate the relationship between self-control and positive life outcomes. Journal of Personality and Social Psychology, 109, 508-525. doi: $10.1037 / \mathrm{pspp} 0000026$

Gallarin, M., \& Alonso-Arbiol, I. (2012). Parenting practices, parental attachment and aggressiveness in adolescence: A predicted model. Journal of Adolescence, 35, 1601-1610. doi:10.1016/j.adolescence.2012.07.002

Garavan, H., Ross, T. J., Murphy, K., Roche, R. A. P., \& Stein, E. A. (2002). Dissociable executive functions in the dynamic control of behavior: Inhibition, error detection, and correction. NeuroImage, 17, 1820-1829. doi:10.1006/ nimg. 2002.1326

Gerson, K. (1985). Hard choices: How women decide about work, career, and motherbood. Berkeley: University of California Press.

*Gibson, C., \& Wright, J. (2001). Low self-control and coworker delinquency: A research note. Journal of Criminal Justice, 29, 483-492. doi:10.1016/S0047-2352(01)00111-8

Giedd, J. N. (2004). Structural magnetic resonance imaging of the adolescent brain. Annals of the New York Academy of Sciences, 1021, 77-85. doi:10.1196/annals.1308.009

Gottfredson, M. R., \& Hirschi, T. (1990). A general theory of crime. Stanford, CA: Stanford University Press.

Grolnick, W. S., McMenamy, J., \& Kurowski, C. (1999). Emotional self-regulation in infancy and toddlerhood. In L. Balter \& C. S. Tamas-LeMonda (Eds.), Child psychology: A handbook of contemporary issues (pp. 3-22). Philadelphia, PA: Psychology Press.

Hagan, J., Simpson, J., \& Gillis, A. R. (1987). Class in the household: A power-control theory of gender and delinquency. American Journal of Sociology, 92, 788-816. doi:10.1086/228583

*Hallquist, M. N., Hipwell, A. E., \& Stepp, S. D. (2015). Poor self-control and harsh punishment in childhood prospectively predict borderline personality symptoms in adolescent girls. Journal of Abnormal Psychology, 124, 549-564. doi: $10.1037 /$ abn0000058

Harden, K. P., Kretsch, N., Mann, F. D., Herzhoff, K., Tackett, J. L., Steinberg, L., . . . Tucker-Drob, E. M. (2017). Beyond dual systems: A genetically-informed, latent factor model of behavioral and self-report measures related to adolescent risk-taking. Developmental Cognitive Neuroscience, 25, 221-234. doi:10.1016/j.dcn.2016.12.007

Hare, T. A., Camerer, C. F., \& Rangel, A. (2009). Self-control in decision-making involves modulation of the vmPFC valuation system. Science, 324, 646-648. doi:10.1126/sci ence. 1168450

Harris, K.M., Halpern, C.T., Whitsel, E., Hussey, J., Tabor, J., Entzel, P., \& Udry, J. R. (2009). The National Longitudinal 
Study of Adolescent to Adult Health: Research Design. Retrieved from http://www.cpc.unc.edu/projects/ addhealth/design

*Hartman, J. D., Patock-Peckham, J. A., Corbin, W. R., Gates, J. R., Leeman, R. F., Luk, J. W., \& King, K. M. (2015). Direct and indirect links between parenting styles, selfconcealment (secrets), impaired control over drinking and alcohol-related outcomes. Addictive Behaviors, 40, 102-108. doi:10.1016/j.addbeh.2014.08.009

*Hartos, J. L., Eitel, P., Haynie, D. L., \& Simons-Morton, B. G. (2000). Can I take the car? Relations among parenting practices and adolescent problem-driving practices. Journal of Adolescent Research, 15, 352-367. doi:10.1177/0743558400153003

*Hay, C. (2001). Parenting, self-control, and delinquency: A test of self-control theory. Criminology, 39, 707-736. doi:10.1111/j.1745-9125.2001.tb00938.x

*Hay, C., \& Meldrum, R. (2010). Bullying victimization and adolescent self-harm: Testing hypotheses from general strain theory. Journal of Youth and Adolescence, 39, 446459. doi:10.1007/s10964-009-9502-0

Hay, C., \& Meldrum, R. (2016). Self-control and crime over the life course. Thousand Oaks, CA: SAGE.

*Hay, C., Meldrum, R. C., \& Piquero, A. R. (2013). Negative cases in the nexus between self-control, social bonds, and delinquency. Youth Violence and Juvenile Justice, 11, 3-25. doi:10.1177/1541204012447959

Hayslett-McCall, K. L., \& Bernard, T. J. (2002). Attachment, masculinity, and self-control: A theory of male crime rates. Theoretical Criminology, 6, 5-33. doi:10.1177/ 136248060200600101

*Hershenberg, R., Davila, J., Yoneda, A., Starr, L. R., Miller, M. R., Stroud, C. B., \& Feinstein, B. A. (2011). What I like about you: The association between adolescent attachment security and emotional behavior in a relationship promoting context. Journal of Adolescence, 34, 1017-1024. doi:10.1016/j.adolescence.2010.11.006

Hoeve, M., Dubas, J. S., Eichelsheim, V. I., van der Laan, P. H., Smeenk, W., \& Gerris, J. R. M. (2009). The relationship between parenting and delinquency: A meta-analyses. Journal of Abnormal Child Psychology, 37, 749-755. doi:10.1007/s10802-009-9310-8

*Hofer, C., Eisenberg, N., \& Reiser, M. (2010). The role of socialization, effortful control, and ego resiliency in French adolescents' social functioning. Journal of Research on Adolescence, 20, 555-582. doi:10.1111/j.15327795.2010.00650.x

Hofstede, G. (2001). Culture's consequences: Comparing values, behaviors, institutions, and organizations across nations (2nd ed.). Thousand Oaks, CA: SAGE.

Hope, T. L., Grasmick, H. G., \& Pointon, L. J. (2003). The family in Gottfredson and Hirschi's general theory of crime: Structure, parenting, and self-control. Sociological Focus, 36, 291-311. doi:10.1080/00380237.2003.10571226

*Houltberg, B. J., Henry, C. S., \& Morris, A. S. (2012). Family interactions, exposure to violence, and emotion regulation: Perceptions of children and early adolescents at risk. Family Relations, 61, 283-296. doi:10.1111/j.17413729.2011.00699.x
*Houltberg, B. J., Morris, A. S., Cui, L., Henry, C. S., \& Criss, M. M. (2016). The role of youth anger in explaining links between parenting and early adolescent prosocial and antisocial behavior. The Journal of Early Adolescence, 36, 297-318. doi:10.1177/0272431614562834

Hox, J. J., Moerbeek, M., \& van de Schoot, R. (2010). Multilevel analysis: Techniques and applications. New York, NY: Routledge.

Hunter, J. E., \& Schmidt, F. L. (1990). Methods of metaanalysis: Correcting error and bias in research findings. Newbury Park, CA: SAGE.

*Hutt, R. L., Wang, Q., \& Evans, G. W. (2009). Relations of parent-youth interactive exchanges to adolescent socioemotional development. Social Development, 18, 785-797. doi:10.1111/j.1467-9507.2008.00518.x

*Intravia, J., Jones, S., \& Piquero, A. R. (2012). The roles of social bonds, personality, and perceived costs: An empirical investigation into Hirschi's "new" control theory. International Journal of Offender Therapy and Comparative Criminology, 56, 1182-1200. doi:10.1177/ $0306624 X 11422998$

*Jabagchourian, J. J., Sorkhabi, N., Quach, W., \& Strage, A. (2014). Parenting styles and practices of Latino parents and Latino fifth graders' academic, cognitive, social, and behavioral outcomes. Hispanic Journal of Behavioral Sciences, 36, 175-194. doi:10.1177/0739986314523289

Jackson, D. (2006). The implication of publication bias for meta-analysis' other parameter. Statistics in Medicine, 25, 2911-2921. doi:10.1002/sim.2293

*Jaffe, M., Gullone, E., \& Hughes, E. K. (2010). The roles of temperamental dispositions and perceived parenting behaviours in the use of two emotion regulation strategies in late childhood. Journal of Applied Developmental Psychology, 31, 47-59. doi:10.1016/j.appdev.2009.07.008

*Janssen, H. J., Eichelsheim, V. I., Deković, M., \& Bruinsma, G. J. (2016). How is parenting related to adolescent delinquency? A between-and within-person analysis of the mediating role of self-control, delinquent attitudes, peer delinquency, and time spent in criminogenic settings. European Journal of Criminology, 13, 169-194. doi:10.1177/1477370815608881

*Jennings, W. G., Higgins, G. E., Tewksbury, R., Gover, A. R., \& Piquero, A. R. (2010). A longitudinal assessment of the victim-offender overlap. Journal of Interpersonal Violence, 25, 2147-2174. doi:10.1177/0886260509354888

*Jiménez-Barbero, J. A., Ruiz-Hernández, J. A., Llor-Esteban, B., \& Waschgler, K. (2016). Influence of attitudes, impulsivity, and parental styles in adolescents' externalizing behavior. Journal of Health Psychology, 21, 122-131. doi:10.1177/1359105314523303

*Jones, S., Lynam, D. R., \& Piquero, A. R. (2015). Substance use, personality, and inhibitors: Testing Hirschi's predictions about the reconceptualization of self-control. Crime $\varepsilon$ Delinquency, 61, 538-558. doi:10.1177/0011128711420109

*Jun, M. H., \& Choi, J. A. (2013). The longitudinal effects of parental monitoring and self-control on depression in Korean adolescents: A multivariate latent growth approach. Children and Youth Services Review, 35, 13271332. doi:10.1016/j.childyouth.2013.05.004 
*Kahn, R. E., Holmes, C., Farley, J. P., \& Kim-Spoon, J. (2015). Delay discounting mediates parent-adolescent relationship quality and risky sexual behavior for low self-control adolescents. Journal of Youth and Adolescence, 44, 16741687. doi:10.1007/s10964-015-0332-y

Karavasilis, L., Doyle, A., \& Markiewicz, D. (2003). Associations between parenting style and attachment to mother in middle childhood and adolescence. International Journal of Behavioral Development, 27, 153-164. doi:10.1080/016 50250244000155

Karreman, A., van Tuijl, C., van Aken, M. A. G., \& Deković, M. (2006). Parenting and self-regulation in preschoolers: A meta-analysis. Infant and Child Development, 15, 561-579. doi:10.1002/icd.478

Kerr, M., \& Stattin, H. (2000). What parents know, how they know it, and several forms of adolescent adjustment: Further support for a reinterpretation of monitoring. Developmental Psychology, 36, 366-380. doi:10.1037/00121649.36.3.366

Kerr, M., Stattin, H., \& Trost, K. (1999). To know you is to trust you: Parents' trust is rooted in child disclosure of information. The Journal of Adolescence, 22, 737-752. doi:10.1006/jado.1999.0266

*Kim, J. E., \& Kim, J. (2015). International note: Teen users' problematic online behavior: Using panel data from South Korea. Journal of Adolescence, 40, 48-53. doi:10.1016/j .adolescence.2015.01.001

*Kim, S., Brody, G. H., \& Murry, V. M. (2003). Longitudinal links between contextual risks, parenting, and youth outcomes in rural African American families. Journal of Black Psychology, 29, 359-377. doi:10.1177/0095798403256887

*Kim-Spoon, J., Farley, J. P., Holmes, C. J., \& Longo, G. S. (2014). Does adolescents' religiousness moderate links between harsh parenting and adolescent substance use? Journal of Family Psychology, 28, 739-748. doi:10.1037/a0037178

*Kim-Spoon, J., Farley, J. P., Holmes, C. J., Longo, G. S., \& McCullough, M. E. (2014). Processes linking parents' and adolescents' religiousness and adolescent substance use: Monitoring and self-control. Journal of Youth and Adolescence, 43, 745-756. doi:10.1007/s10964-013-9998-1

*King, K. M., Lengua, L. J., \& Monahan, K. C. (2013). Individual differences in the development of self-regulation during pre-adolescence: Connections to context and adjustment. Journal of Abnormal Child Psychology, 41, 57-69. doi:10.1007/s10802-012-9665-0

Kochanska, G. (1993). Toward a synthesis of parental socialization and child temperament in early development of conscience. Child Development, 64, 325-347. doi:10.1111/j.1467-8624.1993.tb02913.x

Kochanska, G., Aksan, N., \& Koenig, A. L. (1995). A longitudinal study of the roots of preschoolers' conscience: Committed compliance and emerging internalization. Child Development, 66, 1752-1769. doi:10.1111/14678624.ep9601152100

*Kogan, S. M., Yu, T., Brody, G. H., \& Allen, K. A. (2013). The development of conventional sexual partner trajectories among African American male adolescents. Archives of Sexual Behavior, 42, 825-834. doi:10.1007/s10508-0120025-5
Kopp, C. B. (1982). Antecedents of self-regulation: A developmental perspective. Developmental Psychology, 18, 199-214. doi:10.1037/0012-1649.18.2.199

*Kuhn, E. S., \& Laird, R. D. (2013). Parent and peer restrictions of opportunities attenuate the link between low self-control and antisocial behavior. Social Development, 22, 813-830. doi:10.1111/sode.12028

*Kuther, T. L., \& Fisher, C. B. (1998). Victimization by community violence in young adolescents from a suburban city. The Journal of Early Adolescence, 18, 53-76. doi:10.1177/0272431698018001003

Lakens, D., Hilgard, J., \& Staaks, J. (2016). On the reproducibility of meta-analyses: Six practical recommendations. BMC Psychology, 4, Article 24. doi:10.1186/s40359-0160126-3

Lamb, M. E. (Ed.). (2010). The role of the father in child development (5th ed.). Hoboken, NJ: Wiley.

Lamb, M. E., \& Tamis-LeMonda, C. S. (2004). The role of the father. In M. E. Lamb (Ed.), The role of the father in child development (pp. 1-31). Hoboken, NJ: Wiley.

Lanz, M., Scabini, E., Vermulst, A. A., \& Gerris, J. R. M. (2001). Congruence on child rearing in families with early adolescent and middle adolescent children. International Journal of Behavioral Development, 25, 133-139. doi:10.1080/01650250042000104

*Larsen, J. K., Vermulst, A. A., Eisinga, R., English, T., Gross, J. J., Hofman, E., \& Engels, R. C. (2012). Social coping by masking? Parental support and peer victimization as mediators of the relationship between depressive symptoms and expressive suppression in adolescents. Journal of Youth and Adolescence, 41, 1628-1642. doi:10.1007/ s10964-012-9782-7

*Lee, C.-T., McClernon, F. J., Kollins, S. H., Prybol, K., \& Fuemmeler, B. F. (2013). Childhood economic strains in predicting substance use in emerging adulthood: Mediation effects of youth self-control and parenting practices. Journal of Pediatric Psychology, 38, 1130-1143. doi:10.1093/jpepsy/jst056

*Lee, E. H., Zhou, Q., Eisenberg, N., \& Wang, Y. (2013). Bidirectional relations between temperament and parenting styles in Chinese children. International Journal of Behavioral Development, 37, 57-67. doi:10.1177/0165 025412460795

*Lee, J., Yu, H., \& Choi, S. (2012). The influences of parental acceptance and parental control on school adjustment and academic achievement for South Korean children: The mediation role of self-regulation. Asia Pacific Education Review, 13, 227-237. doi:10.1007/s12564-011-9186-5

*Lengua, L. J. (2006). Growth in temperament and parenting as predictors of adjustment during children's transition to adolescence. Developmental Psychology, 42, 819-832. doi:10.1037/0012-1649.42.5.819

*Lengua, L. J., \& Kovacs, E. A. (2005). Bidirectional associations between temperament and parenting and the prediction of adjustment problems in middle childhood. Journal of Applied Developmental Psychology, 26, 21-38. doi:10.1016/j.appdev.2004.10.001

Lerner, R. M. (2002). Concepts and theories of human development (3rd ed.). Mahwah, NJ: Erlbaum. 
*Lewin-Bizan, S., Bowers, E. P., \& Lerner, R. M. (2010). One good thing leads to another: Cascades of positive youth development among American adolescents. Development and Psychopathology, 22, 759-770. doi:10.1017/S095457 9410000441

*Li, C., Dang, J., Zhang, X., Zhang, Q., \& Guo, J. (2014). Internet addiction among Chinese adolescents: The effect of parental behavior and self-control. Computers in Human Behavior, 41, 1-7. doi:10.1016/j.chb.2014.09.001

*Li, D., Li, X., Wang, Y., Zhao, L., Bao, Z., \& Wen, F. (2013). School connectedness and problematic internet use in adolescents: A moderated mediation model of deviant peer affiliation and self-control. Journal of Abnormal Child Psychology, 41, 1231-1242. doi:10.1007/s10802013-9761-9

*Li, D., Zhang, W., \& Wang, Y. (2015). Parental behavioral control, psychological control and Chinese adolescents' peer victimization: The mediating role of self-control. Journal of Child and Family Studies, 24, 628-637. doi:10.1007/s10826-013-9873-4

*Li, J. B., Delvecchio, E., Lis, A., Nie, Y.-G., \& Di Riso, D. (2015). Parental attachment, self-control, and depressive symptoms in Chinese and Italian adolescents: Test of a mediation model. Journal of Adolescence, 43, 159-170. doi:10.1016/j.adolescence.2015.06.006

*Li, J. B., Delvecchio, E., Miconi, D., Salcuni, S., \& Di Riso, D. (2014). Parental attachment among Chinese, Italian, and Costa Rican adolescents: A cross-cultural study. Personality and Individual Differences, 71, 118-123. doi:10.1016/j.paid.2014.07.036

*Li, X., Li, D., \& Newman, J. (2013). Parental behavioral and psychological control and problematic internet use among Chinese adolescents: The mediating role of self-control. Cyberpsychology, Behavior and Social Networking, 16, 442-447. doi:10.1089/cyber.2012.0293

*Liew, J., Kwok, O., Chang, Y., Chang, B. W., \& Yeh, Y.-C. (2014). Parental autonomy support predicts academic achievement through emotion-related self-regulation and adaptive skills in Chinese American Adolescents. Asian American Journal of Psychology, 5, 214-222. doi:10.1037/a0034787

Lipsey, M. W., \& Wilson, D. B. (2001). Practical meta-analysis. Thousand Oaks, CA: SAGE.

*Liu, Y. L., \& Chang, H. T. (2016). The role of effortful control in the relationships among parental control, intentional self-regulation, and adolescent obedience. Journal of Child and Family Studies, 25, 2435-2446. doi:10.1007/ s10826-016-0405-x

*Loukas, A., \& Roalson, L. A. (2006). Family environment, effortful control, and adjustment among European American and Latino early adolescents. The Journal of Early Adolescence, 26, 432-455. doi:10.1177/0272431606291939

*Loukas, A., Roalson, L. A., \& Herrera, D. E. (2010). School connectedness buffers the effects of negative family relations and poor effortful control on early adolescent conduct problems. Journal of Research on Adolescence, 20, 13-22. doi:10.1111/j.1532-7795.2009.00632.x

Lovejoy, M. C., Graczyk, P. A., O’Hare, E., \& Neuman, G. (2000). Maternal depression and parenting behavior: A meta-analytic review. Clinical Psychology Review, 20, 561-592. doi:10.1016/S0272-7358(98)00100-7

Lynskey, D. P., Winfree, L. T., Esbensen, F.-A., \& Clason, D. L. (2000). Linking gender, monitory group status and family matters to self-control theory: A multivariate analysis of key self-control concepts in a youth-gang context. Juvenile and Family Court Journal, 51, 1-19. doi:10.1111/j.1755-6988.2000.tb00022.x

*Mak, A. S. (1990). Testing a psychosocial control theory of delinquency. Criminal Justice and Behavior, 17, 215-230. doi:10.1177/0093854890017002005

*Mandara, J., \& Pikes, C. L. (2008). Guilt trips and love withdrawal: Does mothers' use of psychological control predict depressive symptoms among African American adolescents? Family Relations, 57, 602-612. doi:10.1111/ j.1741-3729.2008.00526.x

*Marcotte, G., Marcotte, D., \& Bouffard, T. (2002). The influence of familial support and dysfunctional attitudes on depression and delinquency in an adolescent population. European Journal of Psychology of Education, 17, 363-376. doi:10.1007/BF03173591

*McKee, J. R. (2012). The moderation effects of family structure and low self-control. American Journal of Criminal Justice, 37, 356-377. doi:10.1007/s12103-011-9143-1

Meldrum, R. C. (2008). Beyond parenting: An examination of the etiology of self-control. Journal of Criminal Justice, 36, 244-251. doi:10.1016/j.jcrimjus.2008.04.005

*Meldrum, R. C., Barnes, J. C., \& Hay, C. (2015). Sleep deprivation, low self-control, and delinquency: A test of the strength model of self-control. Journal of Youth and Adolescence, 44, 465-477. doi:10.1007/s10964-013-0024-4

*Meldrum, R. C., \& Hay, C. (2012). Do peers matter in the development of self-control? Evidence from a longitudinal study of youth. Journal of Youth and Adolescence, 41, 691-703. doi:10.1007/s10964-011-9692-0

*Meldrum, R. C., Young, J. T. N., Burt, C. H., \& Piquero, A. R. (2013). Maternal versus adolescent reports of self-control: Implications for testing the general theory of crime. Journal of Criminal Justice, 41, 24-32. doi:10.1016/j.jcrim jus.2012.10.003

Meldrum, R. C., Young, J. T. N., Hay, C., \& Flexon, J. L. (2012). Does self-control influence maternal attachment? A reciprocal effect analysis from early childhood through middle adolescence. Journal of Quantitative Criminology, 28, 673-699. doi:10.1007/s10940-012-9173-y

*Meldrum, R. C., Young, J. T. N., \& Lehmann, P. S. (2015). Parental low self-control, parental socialization, young adult low self-control, and offending: A retrospective study. Criminal Justice and Behavior, 42, 1183-1199. doi:10.1177/0093854815595662

Meldrum, R. C., Young, J. T. N., \& Weerman, F. M. (2012). Changes in self-control during adolescence: Investigating the influence of the adolescent peer network. Journal of Criminal Justice, 40, 452-462. doi:10.1016/j.jcrim jus.2012.07.002

Meyer, G. J., Finn, S. E., Eyde, L. D., Kay, G. G., Moreland, K. L., Dies, R. R., . . . Reed, G. M. (2001). Psychological testing and psychological assessment: A review of evidence and issues. 
American Psychologist, 56, 128-165. doi:10.1037/0003066X.56.2.128

Moffitt, T. E., Arseneault, L., Belsky, D., Dickson, N., Hancox, R. J., Harrington, H., . . Caspi, A. (2011). A gradient of childhood self-control predicts health, wealth, and public safety. Proceedings of the National Academy of Sciences, 108, 2693-2698. doi:10.1073/pnas.1010076108

Moher, D., Shamseer, L., Clarke, M., Ghersi, D., Liberati, A., Petticrew, M., . . . Stewart, L. A. (2015). Preferred reporting items for systematic review and meta-analysis protocols (PRISMA-P) 2015 statement. Systematic Reviews, 4, Article 1. doi:10.1186/2046-4053-4-1

*Moilanen, K. L. (2007). The Adolescent Self-Regulatory Inventory: The development and validation of a questionnaire of short-term and long-term self-regulation. Journal of Youth and Adolescence, 36, 835-848. doi:10.1007/ s10964-006-9107-9

*Moilanen, K. L., Rasmussen, K. E., \& Padilla-Walker, L. M. (2015). Bidirectional associations between self-regulation and parenting styles in early adolescence. Journal of Research on Adolescence, 25, 246-262. doi:10.1111/ jora. 12125

*Moilanen, K. L., Shaw, D. S., \& Fitzpatrick, A. (2010). Selfregulation in early adolescence: Relations with motherson relationship quality and maternal regulatory support and antagonism. Journal of Youth and Adolescence, 39, 1357-1367. doi:10.1007/s10964-009-9485-x

*Moon, B., \& Alarid, L. F. (2015). School bullying, low self-control, and opportunity. Journal of Interpersonal Violence, 30, 839-856. doi:10.1177/0886260514536281

*Moon, B., Hwang, H. W., \& McCluskey, J. D. (2011). Causes of school bullying: Empirical test of a general theory of crime, differential association theory, and general strain theory. Crime \& Delinquency, 57, 849-877. doi:10.1177/0011128708315740

*Moon, B., \& Morash, M. (2013). General strain theory as a basis for the design of school interventions. Crime $\mathcal{E}$ Delinquency, 59, 886-909. doi:10.1177/0011128712466949

*Moon, B., Morash, M., \& McCluskey, J. D. (2012). General strain theory and school bullying: An empirical test in South Korea. Crime \& Delinquency, 58, 827-855. doi:10.1177/0011128710364809

*Morris, A. S., \& Age, T. R. (2009). Adjustment among youth in military families: The protective roles of effortful control and maternal social support. Journal of Applied Developmental Psychology, 30, 695-707. doi:10.1016/j .appdev.2009.01.002

Munroe, R. H., Munroe, R. L., Westling, E., \& Rosenberg, J. (1997). Infant experience and late childhood dispositions: An eleven-year follow-up among the Logoli of Kenya. Ethos, 25, 359-372. doi:10.1525/eth.1997.25.3.359

National Research Council. (2015). Investing in the bealth and well-being of young adults. Washington, DC: National Academies Press.

Ng, F. F.-Y., Pomerantz, E. M., \& Deng, C. (2014). Why are Chinese mothers more controlling than American mothers? Child Development, 85, 355-369. doi:10.1111/cdev.12102

*Ngai, S. S. Y. (2015). Parental bonding and character strengths among Chinese adolescents in Hong Kong. International
Journal of Adolescence and Youth, 20, 317-333. doi:10 .1080/02673843.2015.1007879

Ng-Knight, T., Shelton, K. H., Frederickson, N., McManus, I. C., \& Rice, F. (2018). Maternal depressive symptoms and adolescent academic attainment: Testing pathways via parenting and self-control. Journal of Adolescence, 62, 61-69. doi:10.1016/j.adolescence.2017.11.003

*Ng-Knight, T., Shelton, K. H., Riglin, L., McManus, I. C., Frederickson, N., \& Rice, F. (2016). A longitudinal study of self-control at the transition to secondary school: Considering the role of pubertal status and parenting. Journal of Adolescence, 50, 44-55. doi:10.1016/j.adoles cence.2016.04.006

Nickerson, A. B., \& Nagle, R. J. (2005). Parent and peer attachment in late childhood and early adolescence. Journal of Early Adolescence, 25, 223-249. doi:10.1177/0272431604274174

*Nie, Y. G., Li, J. B., \& Vazsonyi, A. T. (2016). Self-control mediates the associations between parental attachment and prosocial behavior among Chinese adolescents. Personality and Individual Differences, 96, 36-39. doi:10.1016/j.paid.2016.02.077

Nigg, J. T. (2017). Annual research review: On the relations among self-regulation, self-control, executive functioning, effortful control, cognitive control, impulsivity, risktaking, and inhibition for developmental psychopathology. Journal of Clinical Psychology and Psychiatry, 58, 361-383. doi:10.1111/jcpp. 12675

*Nofziger, S. (2008). The "cause" of low self-control: The influence of maternal self-control. Journal of Research in Crime \& Delinquency, 45, 191-224. doi:10.1177/00224 27807313708

*O'Connor, M., Sanson, A., Hawkins, M. T., Letcher, P., Toumbourou, J. W., Smart, D., . . . Olsson, C. A. (2011). Predictors of positive development in emerging adulthood. Journal of Youth and Adolescence, 40, 860-874. doi:10.1007/s10964-010-9593-7

O'Connor, T. G. (2002). Annotation: The 'effects' of parenting reconsidered: Findings, challenges, and applications. Journal of Child Psychology and Psychiatry, 43, 555-572. doi:10.1111/1469-7610.00046

*Otterpohl, N., \& Wild, E. (2015). Cross-lagged relations among parenting, children's emotion regulation, and psychosocial adjustment in early adolescence. Journal of Clinical Child E Adolescent Psychology, 44, 93-108. doi: 10.1080/15374416.2013.862802

Oyserman, D., Coon, H. M., \& Kemmelmeier, M. (2002). Rethinking individualism and collectivism: Evaluation of theoretical assumptions and meta-analyses. Psychological Bulletin, 128, 3-72. doi:10.1037//0033-2909.128.1.3

*Özdemir, Y., Vazsonyi, A. T., \& Çok, F. (2013). Parenting processes and aggression: The role of self-control among Turkish adolescents. Journal of Adolescence, 36, 65-77. doi:10.1016/j.adolescence.2012.09.004

*Padilla-Walker, L. M., \& Christensen, K. J. (2011). Empathy and self-regulation as mediators between parenting and adolescents' prosocial behavior toward strangers, friends, and family. Journal of Research on Adolescence, 21, $545-$ 551. doi:10.1111/j.1532-7795.2010.00695.x 
*Padilla-Walker, L. M., \& Coyne, S. M. (2011). "Turn that thing off!" Parent and adolescent predictors of proactive media monitoring. Journal of Adolescence, 34, 705-715. doi:10.1016/j.adolescence.2010.09.002

*Padilla-Walker, L. M., Day, R. D., Dyer, W. J., \& Black, B. C. (2013). "Keep on keeping on, even when it's hard!" Predictors and outcomes of adolescent persistence. The Journal of Early Adolescence, 33, 433-457. doi:10.1177/0272431612449387

Padilla-Walker, L. M., Harper, J. M., \& Jensen, A. C. (2010). Self-regulation as a mediator between sibling relationship quality and early adolescents' positive and negative outcomes. Journal of Family Psychology, 24, 419-428. doi: $10.1037 / \mathrm{a} 0020387$

Pallini, S., Chirumbolo, A., Morelli, M., Baiocco, R., Laghi, F., \& Eisenberg, N. (2018). The relation of attachment security status to effortful self-regulation: A meta-analysis. Psychological Bulletin, 144, 501-531. doi:10.1037/bul0000134

*Park, I. J. K., \& Kim, P. Y. (2012). The role of self-construals in the link between anger regulation and externalizing problems in Korean American adolescents: Testing a moderated mediation model. Journal of Clinical Psychology, 68, 1339-1359. doi:10.1002/jclp.21878

Parke, R. D. (2004). Development in the family. Annual Review of Psychology, 55, 365-399. doi:10.1146/annurev .psych.55.090902.141528

*Patock-Peckham, J. A., Cheong, J., Balhorn, M. E., \& Nagoshi, C. T. (2001). A social learning perspective: A model of parenting styles, self-regulation, perceived drinking control, and alcohol use and problems. Alcoholism: Clinical and Experimental Research, 25, 1284-1292. doi:10.1111/j.1530-0277.2001.tb02349.x

*Patock-Peckham, J. A., \& Morgan-Lopez, A. A. (2006). College drinking behaviors: Mediational links between parenting styles, impulse control, and alcohol-related outcomes. Psychology of Addictive Behaviors, 20, 117-125. doi:10.1037/0893-164X.20.2.117

Patton, G. C., Olsson, C. A., Skirbekk, V., Saffery, R., Wlodek, M. E., Azzopardi, P. S., . . . Sawyer, S. M. (2018). Adolescence and the next generation. Nature, 554, 458466. doi:10.1038/nature25759

Patton, G. C., Sawyer, S. M., Santelli, J. S., Ross, D. A., Afifi, R., Allen, N. B., . . Kakuma, R. (2016). Our future: A Lancet commission on adolescent health and wellbeing. The Lancet, 387, 2423-2478. doi:10.1016/S01406736(16)00579-1

*Pears, K., Capaldi, D. M., \& Owen, L. D. (2007). Substance use risk across three generations: The roles of parent discipline practices and inhibitory control. Psychology of Addictive Behaviors, 21, 373-386. doi:10.1037/0893164X.21.3.373

Plomin, R., DeFries, J. C., \& Loehlin, J. C. (1977). Genotypeenvironment interaction and correlation in the analysis of human behavior. Psychological Bulletin, 84, 309-322. doi:10.1037/0033-2909.84.2.309

Pratt, T. C., \& Cullen, F. T. (2000). The empirical status of Gottfredson and Hirschi's general theory of crime: A metaanalysis. Criminology, 38, 931-964. doi:10.1111/j.1745-91 25.2000.tb00911.x
Prinzie, P., Stams, G. J. J. M., Deković, M., Reijntjes, A. H. A., \& Belsky, J. (2009). The relations between parents' Big Five personality factors and parenting: A meta-analytic review. Journal of Personality and Social Psychology, 97, 351-362. doi:10.1037/a0015823

*Purdie, N., Carroll, A., \& Roche, L. (2004). Parenting and adolescent self-regulation. Journal of Adolescence, 27, 663-676. doi:10.1016/j.adolescence.2004.01.002

Putnam, S. P., Spritz, B. L., \& Stifter, C. A. (2002). Mother-child coregulation during delay of gratification at 30 months. Infancy, 3, 209-225. doi:10.1207/S15327078IN0302_6

*Rathert, J., Fite, P. J., \& Gaertner, A. E. (2011). Associations between effortful control, psychological control and proactive and reactive aggression. Child Psychiatry and Human Development, 42, 609-621. doi:10.1007/s10578011-0236-3

*Raval, V. V., Raval, P. H., \& Deo, N. (2014). Mothers' socialization goals, mothers' emotion socialization behaviors, child emotion regulation, and child socioemotional functioning in urban India. The Journal of Early Adolescence, 34, 229-250. doi:10.1177/0272431613485821

*Ray, J. V., Jones, S., Loughran, T. A., \& Jennings, W. G. (2013). Testing the stability of self-control: Identifying unique developmental patterns and associated risk factors. Criminal Justice and Behavior, 40, 588-607. doi: $10.1177 / 0093854812464222$

R Core Team. (2017). R: A language and environment for statistical computing. Vienna, Austria: R Foundation for Statistical Computing.

*Ren, L., Zhao, J. S., He, N. P., Marshall, I. H., Zhang, H., Zhao, R., \& Jin, C. (2016). Testing for measurement invariance of attachment across Chinese and American adolescent samples. International Journal of Offender Therapy and Comparative Criminology, 60, 964-991. doi:10.1177/ $0306624 X 14566602$

*Rioux, C., Castellanos-Ryan, N., Parent, S., Vitaro, F., Tremblay, R. E., \& Seguin, J. R. (2016). Differential susceptibility to environmental influences: Interactions between child temperament and parenting in adolescent alcohol use. Development and Psychopathology, 28, 265275. doi:10.1017/S0954579415000437

*Rivera-Heredia, M., Guadalupe Martinez-Servin, L., \& Obregon-Velasco, N. (2013). Factors associated with depressive symptomatology in adolescents: The role of family migration and individual, family and social resources. Salud Mental, 36, 115-122.

Romer, D., Duckworth, A. L., Sznitman, S., \& Park, S. (2010). Can adolescents learn self-control? Delay of gratification in the development of control over risk taking. Prevention Science, 11, 319-330. doi:10.1007/s11121-010-0171-8

Rosenthal, R. (1979). The file drawer problem and tolerance for null results. Psychological Bulletin, 86, 638-641. doi:10.1037/0033-2909.86.3.638

Rosenthal, R. (1984). Meta-analytic procedures for social research. Beverly Hills, CA: SAGE.

Rothbaum, F., \& Weisz, J. R. (1994). Parental caregiving and child externalizing behavior in nonclinical samples: A meta-analysis. Psychological Bulletin, 116, 55-74. doi:10.1037/0033-2909.116.1.55 
*Rowe, S. L., Gembeck, Z. M. J., \& Hood, M. (2016). From the child to the neighbourhood: Longitudinal ecological correlates of young adolescents' emotional, social, conduct, and academic difficulties. Journal of Adolescence, 49, 218-231. doi:10.1016/j.adolescence.2016.04.001

*Salvatore, C., \& Markowitz, M. W. (2014). Do life course transitions and social bonds influence male and female offending differently? Gender contrasts and criminality. Deviant Behavior, 35, 628-653. doi:10.1080/01639625 .2013 .867727

Sameroff, A. (2010). A unified theory of development: A dialectic integration of nature and nurture. Child Development, 81, 6-22. doi:10.1111/j.1467-8624.2009.01378.x

*Samuelson, K. W., Krueger, C. E., \& Wilson, C. (2012). Relationships between maternal emotion regulation, parenting, and children's executive functioning in families exposed to intimate partner violence. Journal of Interpersonal Violence, 27, 3532-3550. doi:10.1177/088 6260512445385

Sawyer, S. M., Azzopardi, P. S., Wickremarathne, D., \& Patton, G. C. (2018). The age of adolescence. The Lancet Child \& Adolescent Health, 2, 223-228. doi:10.1016/ S23524642(18)30022-1

*Schreck, C. J., Stewart, E. A., \& Fisher, B. S. (2006). Selfcontrol, victimization, and their influence on risky lifestyles: A longitudinal analysis using panel data. Journal of Quantitative Criminology, 22, 319-340. doi:10.1007/ s10940-006-9014-y

*Schreck, C. J., Wright, R. A., \& Miller, J. M. (2002). A study of individual and situational antecedents of violent victimization. Justice Quarterly, 19, 159-180. doi:10.1080/07418820200095201

*Schwarz, B., Stutz, M., \& Ledermann, T. (2012). Perceived interparental conflict and early adolescents' friendships: The role of attachment security and emotion regulation. Journal of Youth and Adolescence, 41, 1240-1252. doi:10.1007/s10964-012-9769-4

*Shannon, M., Barry, C. M., DeGrace, A., \& DiDonato, T. (2016). How parents still help emerging adults get their homework done: The role of self-regulation as a mediator in the relation between parent-child relationship quality and school engagement. Journal of Adult Development, 23, 36-44. doi:10.1007/s10804-015-9219-0

*Shin, S. H., Cook, A. K., Morris, N. A., McDougle, R., \& Groves, L. P. (2016). The different faces of impulsivity as links between childhood maltreatment and young adult crime. Preventive Medicine, 88, 210-217. doi:10.1016/j .ypmed.2016.03.022

Silverman, I. W., \& Ragusa, D. M. (1990). Child and maternal correlates of impulse control in 24-month-old children. Genetic, Social, and General Psychology Monographs, 116, 437-473.

*Simons, L. G., Simons, R. L., Landor, A. M., Bryant, C. M., \& Beach, S. R. H. (2014). Factors linking childhood experiences to adult romantic relationships among African Americans. Journal of Family Psychology, 28, 368-379. doi: $10.1037 / \mathrm{a} 0036393$

*Simons, L. G., Sutton, T. E., Simons, R. L., Gibbons, F. X., \& Murry, V. M. (2016). Mechanisms that link parenting practices to adolescents' risky sexual behavior: A test of six competing theories. Journal of Youth and Adolescence, 45, 255-270. doi:10.1007/s10964-015-0409-7

*Simons, R. L., Simons, L. G., Chen, Y. F., Brody, G. H., \& Lin, K. H. (2007). Identifying the psychological factors that mediate the association between parenting practices and delinquency. Criminology, 45, 481-517. doi:10.1111/ j.1745-9125.2007.00086.x

Situ, Q. M., Li, J. B., \& Dou, K. (2016). Reexamining the linear and U-shaped relationships between self-control and emotional and behavioral problems. Asian Journal of Social Psychology, 19, 177-185. doi:10.1111/ajsp.12118

Slagt, M., Dubas, J. S., Deković, M., \& van Aken, M. A. G. (2016). Differences in sensitivity to parenting depending on child temperament: A meta-analysis. Psychological Bulletin, 142, 1068-1110. doi:10.1037/bul0000061

Smetana, J. G. (2017). Current research on parenting styles, dimensions, and beliefs. Current Opinion in Psychology, 15, 19-25. doi:10.1016/j.copsyc.2017.02.012

Smetana, J. G., Campione-Barr, N., \& Metzger, A. (2006). Adolescent development in interpersonal and societal contexts. Annual Review of Psychology, 57, 255-284. doi:10.1146/annurev.psych.57.102904.190124

*Smith-Bynum, M. H., \& Brody, G. H. (2005). Coping behaviors, parenting, and perceptions of children's internalizing and externalizing problems in rural African American mothers. Family Relations, 54, 58-71. doi:10.1111/j.01976664.2005.00006.x

Song, H., Thompson, R. A., \& Ferrer, E. (2009). Attachment and self-evaluation in Chinese adolescents: Age and gender differences. Journal of Adolescence, 32, 1267-1286. doi:10.1016/j.adolescence.2009.01.001

Sroufe, A. L. (1996). Emotional development: The organization of emotional life in the early years. New York, NY: Cambridge University Press.

Steinberg, L. (2004). Risk-taking in adolescence: What changes, and why? Annals of the New York Academy of Sciences, 1021, 51-58. doi:10.1196/annals.1308.005

Steinberg, L., \& Morris, A. S. (2001). Adolescent development. Annual Review of Psychology, 52, 83-110. doi:10.1146/ annurev.psych.52.1.83

Steinberg, L., \& Silk, J. S. (2002). Parenting adolescents. In M. H. Bornstein (Ed.), Handbook of parenting (Vol. 1, 2nd ed., pp. 103-133). Mahwah, NJ: Erlbaum.

*Stevens, D. L., \& Hardy, S. A. (2013). Individual, family, and peer predictors of violence among Samoan adolescents. Youth \& Society, 45, 428-449. doi:10.1177/00441 18X11424756

*Stevens, E. N., Bardeen, J. R., \& Murdock, K. W. (2015). Parenting behaviors and anxiety in young adults: Effortful control as a protective factor. Journal of Individual Differences, 36, 170-176. doi:10.1027/1614-0001/a000169

Strand, P. S. (2002). Coordination of maternal directives with preschoolers' behavior: Influence of maternal coordination training on dyadic activity and child compliance. Journal of Clinical Child and Adolescence Psychology, 31, 6-15. doi:10.1207/153744202753441620

Sutton, A. J., Duval, S. J., Tweedie, R. L., Abrams, K. R., \& Jones, D. R. (2000). Empirical assessment of effect of 
publication bias on meta-analyses. BMJ, 320, 1574-1577. doi:10.1136/bmj.320.7249.1574

Tangney, J. P., Baumeister, R. F., \& Boone, A. L. (2004). High self-control predicts good adjustment, less pathology, better grades, and interpersonal success. Journal of Personality, 72, 271-324. doi:10.1111/j.00223506.2004.00263.x

*Tarantino, N., Lamis, D. A., Ballard, E. D., Masuda, A., \& Dvorak, R. D. (2015). Parent-child conflict and drug use in college women: A moderated mediation model of self-control and mindfulness. Journal of Counseling Psychology, 62, 303-313. doi:10.1037/cou0000013

*Teasdale, B., \& Silver, E. (2009). Neighborhoods and selfcontrol: Toward an expanded view of socialization. Social Problems, 56, 205-222. doi:10.1525/sp.2009.56.1.205

*Tiberio, S. S., Capaldi, D. M., Kerr, D. C. R., Bertrand, M., Pears, K. C., \& Owen, L. (2016). Parenting and the development of effortful control from early childhood to early adolescence: A transactional developmental model. Development and Psychopathology, 28, 837-853. doi:10.1017/S0954579416000341

Torgerson, C. J. (2006). Publication bias: The Achilles' heel of systematic review? British Journal of Educational Studies, 54, 89-102. doi:10.1111/j.1467-8527.2006.00332.x

*Turner, M. G., Piquero, A. R., \& Pratt, T. C. (2005). The school context as a source of self-control. Journal of Criminal Justice, 33, 327-339. doi:10.1016/j.jcrimjus.2005.04.003

United Nations Children's Fund. (2011). The state of the world's children 2011: Adolescence: An age of opportunity. Retrieved from https://www.unicef.org/sowc2011

*Unnever, J. D., Cullen, F. T., \& Agnew, R. (2006). Why is "bad" parenting criminogenic? Implications from rival theories. Youth Violence and Juvenile Justice, 4, 3-33. doi:10.1177/1541204005282310

Van den Noortgate, W., López-López, J. A., Marín-Martínez, F., \& Sánchez-Meca, J. (2013). Three-level meta-analysis of dependent effect sizes. Behavior Research Methods, 45 , 576-594. doi:10.3758/s13428-012-0261-6

*van der Voort, A., Linting, M., Juffer, F., BakermansKranenburg, M. J., \& van Ijzendoorn, M. H. (2013). Delinquent and aggressive behaviors in early-adopted adolescents: Longitudinal predictions from child temperament and maternal sensitivity. Children and Youth Services Review, 35, 439-446. doi:10.1016/j.childyouth.2012.12.008

*van Durme, K., Braet, C., \& Goossens, L. (2015). Insecure attachment and eating pathology in early adolescence: Role of emotion regulation. The Journal of Early Adolescence, 35, 54-78. doi:10.1177/0272431614523130

*Vazsonyi, A. T., \& Belliston, L. M. (2007). The family $\rightarrow$ low self-control $\rightarrow$ deviance: A cross-cultural and crossnational test of self-control theory. Criminal Justice and Behavior, 34, 505-530. doi:10.1177/0093854806292299

*Vazsonyi, A. T., \& Huang, L. (2010). Where self-control comes from: On the development of self-control and its relationship to deviance over time. Developmental Psychology, 46, 245-257. doi:10.1037/a0016538

Vazsonyi, A. T., \& Jiskrova, G. K. (2017). On the development of self-control and deviance from preschool to middle adolescence. Journal of Criminal Justice, 56, 60-69. doi:10.1016/j.jcrimjus.2017.08.005

*Vazsonyi, A. T., Jiskrova, G. K., Ksinan, A. J., \& Blatny, M. (2016). An empirical test of self-control theory in Roma adolescents. Journal of Criminal Justice, 44, 66-76.

*Vazsonyi, A. T., \& Klanjsek, R. (2008). A test of self-control theory across different socioeconomic strata. Justice Quarterly, 25, 101-131. doi:10.1080/07418820801954571

Vazsonyi, A. T., Mikuška, J., \& Kelley, E. L. (2017). It's time: A meta-analysis on the self-control-deviance link. Journal of Criminal Justice, 48, 48-63. doi:10.1016/j.jcrim jus.2016.10.001

*Vazsonyi, A. T., Trejos-Castillo, E., \& Huang, L. (2006). Risky sexual behaviors, alcohol use, and drug use: A comparison of Eastern and Western European adolescents. Journal of Adolescent Health, 39, 753.e1-753.e11. doi:10.1016/j.jadohealth.2006.05.008

*Veenstra, R., Lindenberg, S., Oldehinkel, A. J., De Winter, A. F., \& Ormel, J. (2006). Temperament, environment, and antisocial behavior in a population sample of preadolescent boys and girls. International Journal of Behavioral Development, 30, 422-432. doi:10.1177/0165025406071490

*Veenstra, R., Lindenberg, S., Tinga, F., \& Ormel, J. (2010). Truancy in late elementary and early secondary education: The influence of social bonds and self-controlthe TRAILS study. International Journal of Behavioral Development, 34, 302-310. doi:10.1177/0165025409347987

*Veenstra, R., Lindenberg, S., Verhulst, F. C., \& Ormel, J. (2009). Childhood-limited versus persistent antisocial behavior: Why do some recover and others do not? The TRAILS study. The Journal of Early Adolescence, 29, 718742. doi:10.1177/0272431608325501

*Vera, E. P., \& Moon, B. (2013). An empirical test of low selfcontrol theory: Among Hispanic youth. Youth Violence and Juvenile Justice, 11, 79-93. doi:10.1177/1541204012441628

Viechtbauer, W. (2010). Conducting meta-analyses in R with the metafor package. Journal of Statistical Software, 36, 1-48. doi:10.18637/jss.v036.i03

*Walters, G. D. (2015). Early childhood temperament, maternal monitoring, reactive criminal thinking, and the origin(s) of low self-control. Journal of Criminal Justice, 43, 369-376. doi:10.1016/j.jcrimjus.2015.07.001

*Walters, G. D., \& DeLisi, M. (2013). Antisocial cognition and crime continuity: Cognitive mediation of the past crimefuture crime relationship. Journal of Criminal Justice, 41, 135-140. doi:10.1016/j.jcrimjus.2012.12.004

*Walton, A., \& Flouri, E. (2010). Contextual risk, maternal parenting and adolescent externalizing behaviour problems: The role of emotion regulation. Child Care Health Development, 36, 275-284. doi:10.1111/j.13652214.2009.01065.x

*Wang, M.-T., Brinkworth, M., \& Eccles, J. (2013). Moderating effects of teacher-student relationship in adolescent trajectories of emotional and behavioral adjustment. Developmental Psychology, 49, 690-705. doi:10.1037/ a0027916

*Watts, S. J., \& McNulty, T. L. (2016). Genes, parenting, selfcontrol, and criminal behavior. International Journal 
of Offender Therapy and Comparative Criminology, 60, 469-491. doi:10.1177/0306624X14553813

Weisz, J. R., Kuppens, S., Ng, M. Y., Eckshtain, D., Ugueto, A. M., Vaughn-Coaxum, R., . . . Weersing, V. R. (2017). What five decades of research tells us about the effects of youth psychological therapy: A multilevel meta-analysis and implications for science and practice. American Psychologist, 72, 79-117. doi:10.1037/a0040360

*Wentzel, K. R., Feldman, S. S., \& Weinberger, D. A. (1991). Parental child rearing and academic achievement in boys: The mediational role of social-emotional adjustment. The Journal of Early Adolescence, 11, 321-399. doi:10.1177/0272431691113002

Willems, Y. E., Boesen, N., Li, J., Finkenauer, C., \& Bartels, M. (2019). The heritability of self-control: A meta-analysis. Neuroscience \& Biobehavioral Reviews, 100, 324-334. doi:10.1016/j.neubiorev.2019.02.012

Willems, Y. E., de Zeeuw, E. L., van Beijsterveldt, C. E., Boomsma, D. I., Bartels, M., \& Finkenauer, C. (2019). Out of control: Examining the association between family conflict and self-control in adolescence in a genetically sensitive design. Journal of the American Academy of Child \& Adolescent Psychiatry. Advance online publication. doi:10.1016/j.jaac.2019.02.017

Willems, Y. E., Dolan, C. V., van Beijsterveldt, C. E. M., de Zeeuw, E. L., Boomsma, D. I., Bartels, M., \& Finkenauer, C. (2018). Genetic and environmental influences on selfcontrol: Assessing self-control with the ASEBA Self-Control Scale. Behavior Genetics, 48, 135-146. doi:10.1007/ s10519-018-9887-1

*Wills, T. A., Cleary, S., Filer, M., Shinar, O., Mariani, J., \& Spera, K. (2001). Temperament related to early-onset substance use: Test of a developmental model. Prevention Science, 2, 145-163. doi:10.1023/A:1011558807062

*Wills, T. A., Gibbons, F. X., Gerrard, M., Murry, V. M., \& Brody, G. H. (2003). Family communication and religiosity related to substance use and sexual behavior in early adolescence: A test for pathways through self-control and prototype perceptions. Psychology of Addictive Behaviors, 17, 312-323. doi:10.1037/0893-164X.17.4.312

*Wills, T. A., Resko, J. A., Ainette, M. G., \& Mendoza, D. (2004). Role of parent support and peer support in adolescent substance use: A test of mediated effects. Psychology of Addictive Behaviors, 18, 122-134. doi:10.1037/0893164X.18.2.122

*Wilson, A. C., Lengua, L. J., Meltzoff, A. N., \& Smith, K. A. (2010). Parenting and temperament prior to September 11,2001 , and parenting specific to $9 / 11$ as predictors of children's posttraumatic stress symptoms following 9/11. Journal of Clinical Child and Adolescent Psychology, 39, 445-459. doi:10.1080/15374416.2010.486317

*Wong, M. M. (2008). Perceptions of parental involvement and autonomy support: Their relations with selfregulation, academic performance, substance use and resilience among adolescents. North American Journal of Psychology, 10, 497-518.

World Health Organization. (2017). Adolescent health. Retrieved from http://www.who.int/topics/adolescent_health/en

*Wright, B. R. E., Caspi, A., Moffitt, T. E., \& Silva, P. A. (1999). Low self-control, social bonds, and crime: Social causation, social selection, or both? Criminology, 37, 479-514. doi:10.1111/j.1745-9125.1999.tb00494.x

*Yap, M. B., Schwartz, O. S., Byrne, M. L., Simmons, J. G., \& Allen, N. B. (2010). Maternal positive and negative interaction behaviors and early adolescents' depressive symptoms: Adolescent emotion regulation as a mediator. Journal of Research on Adolescence, 20, 1014-1043. doi:10.1111/j.1532-7795.2010.00665.x

*You, S., \& Kim, A. Y. (2016). Understanding aggression through attachment and social emotional competence in Korean middle school students. School Psychology International, 37, 255-270. doi:10.1177/0143034316631039

*Yu, J. J. (2010). Reciprocal influences between parental knowledge and adolescent self-control: A cross-sequential nationwide study of Korean youth. Journal of Family Psychology, 24, 458-467. doi:10.1037/a0020425

*Zhang, H., Li, D., \& Li, X. (2015). Temperament and problematic internet use in adolescents: A moderated mediation model of maladaptive cognition and parenting Styles. Journal of Child and Family Studies, 24, 1886-1897. doi:10.1007/s10826-014-9990-8

*Zhu, H., Luo, X., Cai, T., Li, Z., \& Liu, W. (2014). Selfcontrol and parental control mediate the relationship between negative emotions and emotional eating among adolescents. Appetite, 82, 202-207. doi:10.1016/j .appet.2014.06.106 